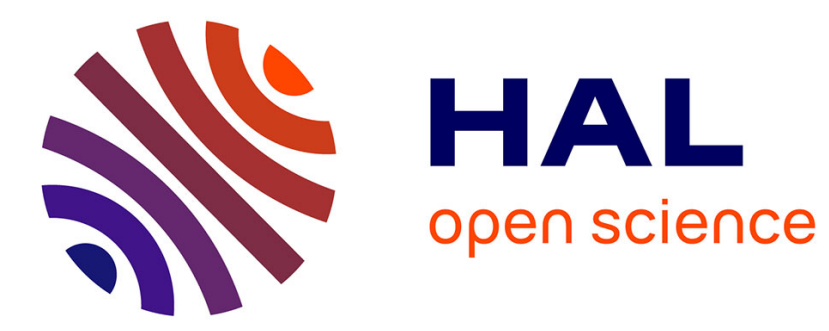

\title{
Equitable location of facilities in a region with probabilistic barriers to travel
}

M. Amiri Aref, Reza Zanjirani Farahani, Michael Hewitt, W. Klibi

\section{To cite this version:}

M. Amiri Aref, Reza Zanjirani Farahani, Michael Hewitt, W. Klibi. Equitable location of facilities in a region with probabilistic barriers to travel: .. Transportation Research Part E: Logistics and Transportation Review, 2019. hal-03421936

\section{HAL Id: hal-03421936 https://hal.science/hal-03421936}

Submitted on 21 Dec 2021

HAL is a multi-disciplinary open access archive for the deposit and dissemination of scientific research documents, whether they are published or not. The documents may come from teaching and research institutions in France or abroad, or from public or private research centers.
L'archive ouverte pluridisciplinaire HAL, est destinée au dépôt et à la diffusion de documents scientifiques de niveau recherche, publiés ou non, émanant des établissements d'enseignement et de recherche français ou étrangers, des laboratoires publics ou privés. 
Biosynthesis of $\mathrm{TiO}_{2}$ nanoparticles and their application for treatment of brain injuryAn in-vitro toxicity study towards central nervous system

Yun Sun, Si Wang, Jin Zheng*

Department of Neurosurgery, Union Hospital, Tongji Medical College, Huazhong University of Science and Technology, Wuhan, China

Corresponding author: Jin Zheng, Department of Neurosurgery, Union Hospital, Tongji Medical College, Huazhong University of Science and Technology, No.1277, Jiefang Street, Jianghan District, Wuhan 430022, China, Email: jinzheng687@yahoo.com 


\begin{abstract}
In the current study, a facile green synthesis of $\mathrm{TiO}_{2}$ nanoparticles (NPs) utilizing the leaf extract of Lippia citriodora as a stabilizing and reducing agent was reported. The prepared $\mathrm{TiO}_{2}$ NPs were studied using XRD, UV, HRTEM, FTIR, Raman and EDS analysis. TEM analysis confirmed that the nanoparticles size is in $20-40 \mathrm{~nm}$ range. FTIR and UVVisible spectra represented the $\mathrm{TiO}_{2}$ NPs formation. Similarly, the analysis of XRD and EDS validated the crystalline rutile structure of $\mathrm{TiO}_{2} \mathrm{NPs}$ formed. In addition, this investigation was shown to examine the $\mathrm{TiO}_{2} \mathrm{NPs}$ toxicity on the CNS central nervous system in vitro. In the extracted cell cultures from the rats ECB embryonic cortical brain, substantial decline in the neuroblasts has been noticed once after incubating with $\mathrm{TiO}_{2} \mathrm{NPs}$ for $24 \mathrm{~h}$ to $96 \mathrm{~h}$ ( 5 to 20 $\mu \mathrm{g} / \mathrm{ml})$. This study also demonstrates the decline of neuroblast proliferation. In the conclusion, our investigation illustrated evidently the $\mathrm{TiO}_{2} \mathrm{NPs}$ toxic effect on the neuronal cells and rat brain also mentioned about toxicity effects of $\mathrm{TiO}_{2}$ which were not yet described, for example the decline in vitro neuroblast proliferation.
\end{abstract}


Keywords: $\mathrm{TiO}_{2} \mathrm{NPs}$, brain injury, neuroblasts, astrocytes

\section{Introduction}

In recent days, it has been identified that there is an enormous interest on the study of nanomaterials. Nano biotechnology is a growing technology that deals with materials of nano meter sized in various scientific fields like material science, chemistry, physics, biotechnology and nanotechnology. Apart from various physical and chemical methods which are fixed for metallic nanoparticles preparation, nano biotechnology also plays as a prominent method, for the evaluation of safe, eco-friendly and hygienic methods for synthesis of metallic nanoparticles (NPs) [1]. Recently, green synthesis of NPs has grabbed significant attention due to the promising requirement in harmless chemicals, antiviral, antibacterial, diagnostics, renewable materials, aimed delivery of drug, environmental responsive solvents and anticancer [2].

Previous studies explained that the cause of larger particles production, which absorbs more energy, is due to the presence of chemical reducing agent. In addition, it was communicated that other side effects were because of the environment unfriendly methods. Moreover, the NPs, which are chemically synthesized, were reported to exhibit added agglomeration and less stability [3-5]. Subsequently, it is necessary to advance a nature friendly mechanism that produces controllable size of dispersible and stable NPs, which also absorbs little energy.

$\mathrm{TiO}_{2}$ Titanium dioxide is a precious semi-conducting material of transition metal oxide which shows exceptional features like simple control, non-toxicity, reduced cost and better resistance to the chemical corrosion, which helps to use in chemical sensors, solar 
cells, and also in applications of environmental distillation [6]. These NPs contains good properties like optical, magnetic and electrical, which vary from many other complements [7]. Crystalline and amorphous forms were produced by Titanium dioxide and occurs primarily in three polymorphous crystalline forms that are brookite, rutile and anatase [8]. Various studies have explained the use of biomolecules from plants that are having medicinal value like Psidium guajava, Calotropis gigantean, Ageratina alttissima L, Aloe barbadensis Miller, Psidium guajava, Vitex negundo, Vigna unguiculata, Curcuma longa, Moringa oleifera and Eclipta prostrata for the preparation of $\mathrm{TiO}_{2} \mathrm{NPs}$. Using plants large amount of NPs can be prepared effortlessly and majority product which synthesized is not harmful.

In the present work, we showed the green synthesis of $\mathrm{TiO}_{2}$ nanoparticles (NPs) utilizing the leaf extract of Lippia citriodora as a stabilizing and reducing agent. Further, we examined the $\mathrm{TiO}_{2} \mathrm{NPs}$ toxicity on the CNS central nervous system in vitro. Our investigation illustrated evidently the $\mathrm{TiO}_{2} \mathrm{NPs}$ toxic effect on the neuronal cells and rat brain also mentioned about toxicity effects of $\mathrm{TiO}_{2}$, which were not yet described.

\section{Materials and methods}

\section{Materials}

Titanium tetrachloride $\left(\mathrm{TiCl}_{4}\right.$, analytical grade, $99.9 \%$ purity), other reagents and solvents were obtained from Sigma-Aldrich.

Preparation of Lippia citriodora leaf extract and synthesis of $\mathrm{TiO}_{2} \mathrm{NPS}$

The healthy fresh leaves of Lippia citriodora were thoroughly cleaned for removing dust particles using tap water for about 10 min and then briefly washed with deionized water. 
For preparing the broth solution of plant leaf, $10 \mathrm{~g}$ of finely, cut and washed leaves were taken in a $250 \mathrm{ml}$ of Erlenmeyer flask along with deionized water of $100 \mathrm{ml}$ and then the solution was boiled at $60^{\circ} \mathrm{C}$ for about $5 \mathrm{~min}$. Whatman no. 1 filter paper was used for filtering the solution after boiling. For $\mathrm{TiO}_{2} \mathrm{NPs}$ synthesis, $\mathrm{TiCl}_{4}$ solution $(5 \mathrm{mM})$ of $80 \mathrm{ml}$ was taken in an Erlenmeyer flask and stirred for about 2 hours. $20 \mathrm{ml}$ of Lippia citriodora aqueous extract was mixed to $\mathrm{TiCl}_{4}$ solution $(5 \mathrm{mM})$ of $80 \mathrm{ml}$ at normal room temperature for about 15 min later the final solution turns to pink colour.

\section{In vitro experiments}

\section{Cell cultures}

All the experiments were performed with approval from ethical committee as per university and ethical committee guidelines. Wistar Han (Gravid rats) were sedated with Nembutal (125 mg/kg) an IP (intraperitoneal) injection. From the uterus embryos (i.e E18E19) are detached. The embryos are amputated, and craniums are positioned instantly in the chilled HBSS (Hank's Balanced Salt Solution). Hemisphaerium cerebri were detached and sited in a germ-free 100 -mm plate comprising of an additional chilled medium dissection (HBSS). Underneath a dividing microscope, brain hemispheres are parted and Cortex cerebri was cautiously separated eradicating meninges and the midbrain. Cerebral cortex models were shifted in a sterilized tube comprising HBSS of $2 \mathrm{ml}$ and distanced mechanically.

Post cell density assessment by means of a haemocytometer (Brüker), suspensions of cell were weakened and coated at density of $7 \times 10^{4} / 1.13 \mathrm{~cm}^{2}$ on disinfected $12 \mathrm{~mm}$ wide circular glass coverslips initially covered with polylysine in $24-$ well plates. In an incubator cultures were sited at about $37{ }^{\circ} \mathrm{C}$ along with moist atmosphere at 5\% Carbon dioxide. Cells are nourished with replenished (1\% FBS) medium twice a week. 
Exposure of culture with $\mathrm{TiO}_{2} \mathrm{NPS}$

A bulk quantity of about $2.5-10 \mu \mathrm{l}$ of $\mathrm{TiO}_{2}$ standard suspension $(2 \mathrm{mg} / \mathrm{ml})$ was supplemented for each (Neurobasal/1\% FBS) culture medium $\mathrm{ml}$ so that final concentrations of $(20,15,10$ or $5 \mu \mathrm{g} / \mathrm{ml})$ can be obtained in the well plates. The cultures are uncovered for diverse periodical intervals of 96, 72, 24, 18 and 6 hours. Same quantity of 2.5-10 $\mu$ l of PBS were supplemented in control cultures for each $\mathrm{ml}$ of culture medium. During the period of incubation, the culture medium was not altered.

\section{Evaluation of cell proliferation}

Cell proliferation was assessed using immunocytochemical recognition of (BrdU) 5Bromo-2'-deoxyuridine in culture. Fleetingly, culture cells were treated to $3 \mu \mathrm{g} / \mathrm{ml} \mathrm{BrdU}$ over a period of $2 \mathrm{~h}$ prior to cell fixation. Post fixation for 15 mins in $4 \%$ paraformaldehyde, the culture portions were washed in purified $\mathrm{H}_{2} \mathrm{O}$ and preserved for about 30 min along with the solution of $3 \mathrm{M} \mathrm{HCl}$ at $60{ }^{\circ} \mathrm{C}$. Post cleaning with PBS, pre incubation of culture cells was done in PBS Buffer for about 20 min along with a solution of $0.01 \%$ casein. Subsequently, a rat monoclonal anti-BrdU antibody (1:20) was used to incubate cells for about $1 \mathrm{hr}$ at the room temperature. This stage was shadowed by a contact of $30 \mathrm{~min}$ to peroxidase/anti-mouse complexes (ImmPressTM Reagent Kit; CA, Burlingame, Vector). Disclosure of bound peroxidase action was achieved by incubating in PBS along with solution of $0.05 \%$ DAB (3.3'-diaminobenzidine) and $0.02 \% \mathrm{H}_{2} \mathrm{O}_{2}$. Lastly, culture cells were mounted in everlasting medium after getting counter-stained with Mayer's hemalun. The sum of S-phase cells were calculated on 50 microscopic areas chosen at random for each slide at 400X magnification. For every time of revelation to NPs and for every $\mathrm{TiO}_{2}$ concentration, trials were done on 4 self-regulating cultures, 4 no-treated cultures were assessed succeeding a parallel process and 
were cast-off as controls. For every trial state, the mean was planned on four self-regulating cultures and facts offered as histogram $+/$ - SEM.

\section{Characterization of $\mathrm{TiO}_{2} \mathrm{NPs}$}

The green reduction of $\mathrm{TiO}_{2} \mathrm{NPs}$ was supervised by sampling the solution mixture at uniform intervals, and UV-vis spectra was used for scanning the absorption maxima at a wavelength of 200-700 $\mathrm{nm}$ in Schimadzu spectrophotometer 1601 operated at $1 \mathrm{~nm}$ resolution. The reaction solution was centrifuged at 10,000 rpm at room temperature (i.e 25 ${ }^{\circ} \mathrm{C}$ ) for about $45 \mathrm{~min}$. The final product was dispersed in the deionized water, and then Millipore filter of $0.22 \mathrm{~mm}$ was used for filtering. The produced NPs were utilized for Dynamic light scattering (DLS), High- resolution transmission electron microscopy (HRTEM), Energy dispersive X-ray spectroscopy (EDX), X-ray diffraction (XRD), Zetapotential and Fourier transform infrared (FTIR).

The $\mathrm{TiO}_{2}$ nanoparticles XRD measurements were performed on the respective films of the solutions drop covered on to crystal substrates on 1830 Phillips PW apparatus functioning at $40 \mathrm{kV}$ voltage and $30 \mathrm{~mA}$ current with CuKa1 radioactivity. Classification involved FTIR investigation of the $\mathrm{TiO}_{2}$ nanoparticles dried precipitate, was performed by examining it in 500-4000 $\mathrm{cm}^{-1}$ of range and $4 \mathrm{~cm}^{-1}$ of resolution. Perkin Elmer Spectrum One apparatus is used for performing these measurements in the mode of diffuse reflectance in the $\mathrm{KBr}$ pellets ( $\mathrm{KBr}$ powder is mixed with pellets and dried properly for pelletizing). The morphology and size of green synthesized nanoparticles were noticed by the help of HRTEM investigation. The $\mathrm{TiO}_{2}$ nanoparticles were placed on the copper grid which was carboncoated and then dried in the air before to the microscopic analysis (model 3010 JEOL)

functioned at $0.0251 \AA$ wavelength and $200 \mathrm{keV}$ accelerating voltage. The classification of 
Zeta potential and hydrodynamic size of $\mathrm{TiO}_{2}$ nanoparticles in solution was known using Dynamic light scattering (DLS) (carried out on a Malvern Apparatuses which was Zeta sizer, Nano-ZS).

\section{Results and discussion}

$\mathrm{TiO}_{2} \mathrm{NPs}$ prepared using Lippia citriodora leaf extract exhibited a strong UV-Visible optical absorbance peak at $400 \mathrm{~nm}$, indicated the formation of $\mathrm{TiO}_{2} \mathrm{NPs}_{\text {(Fig.1). FTIR studies }}$ were performed to know the high purity of formed product. The FTIR results (as shown in Fig. 2) of these NPs represented the bands corresponding to only $\mathrm{TiO}_{2}$. The band detected at $590 \mathrm{~cm}^{-1}$ is because of the Ti-O-O bond vibration. The FTIR results firmly recommends the existence of Ti-O bonds and the nonexistence of groups $\mathrm{OH}$ and peroxo in the ultimate product. The final $\mathrm{TiO}_{2}$ nanoparticles produced by this process are of top quality which can also be utilized in future applications. An intense broad peak at about $3430 \mathrm{~cm}^{-1}$ in the FTIR result can be allocated to the bond $\mathrm{N}-\mathrm{H}$ extending frequency rising from the peptide bonds existing in the proteins of the Lippia citriodora green synthesis by means of $\mathrm{TiO}_{2}$. An intense absorption band at $1779 \mathrm{~cm}^{-1}$ is the representation of irregular $\mathrm{C}=\mathrm{O}$ stretch vibration of anhydride group. The change in the region of carbonyl (1600-1800 $\left.\mathrm{cm}^{-1}\right)$ was observed using FTIR and the presence of characteristic peaks at $1639 \mathrm{~cm}^{-1}$ and $1779 \mathrm{~cm}^{-1}$ were corresponding to amide II and amide I, also an intense decline in the relative peaks intensity (distinctive feature of the regular $\mathrm{C}=\mathrm{O}$ broadening vibrations from the groups of maleic anhydride). New peaks at $1639 \mathrm{~cm}^{-1}$ and $1779 \mathrm{~cm}^{-1}$ represented the amide II and amide I shows the existence of the residues of amino acid from Lippia citriodora extract. 


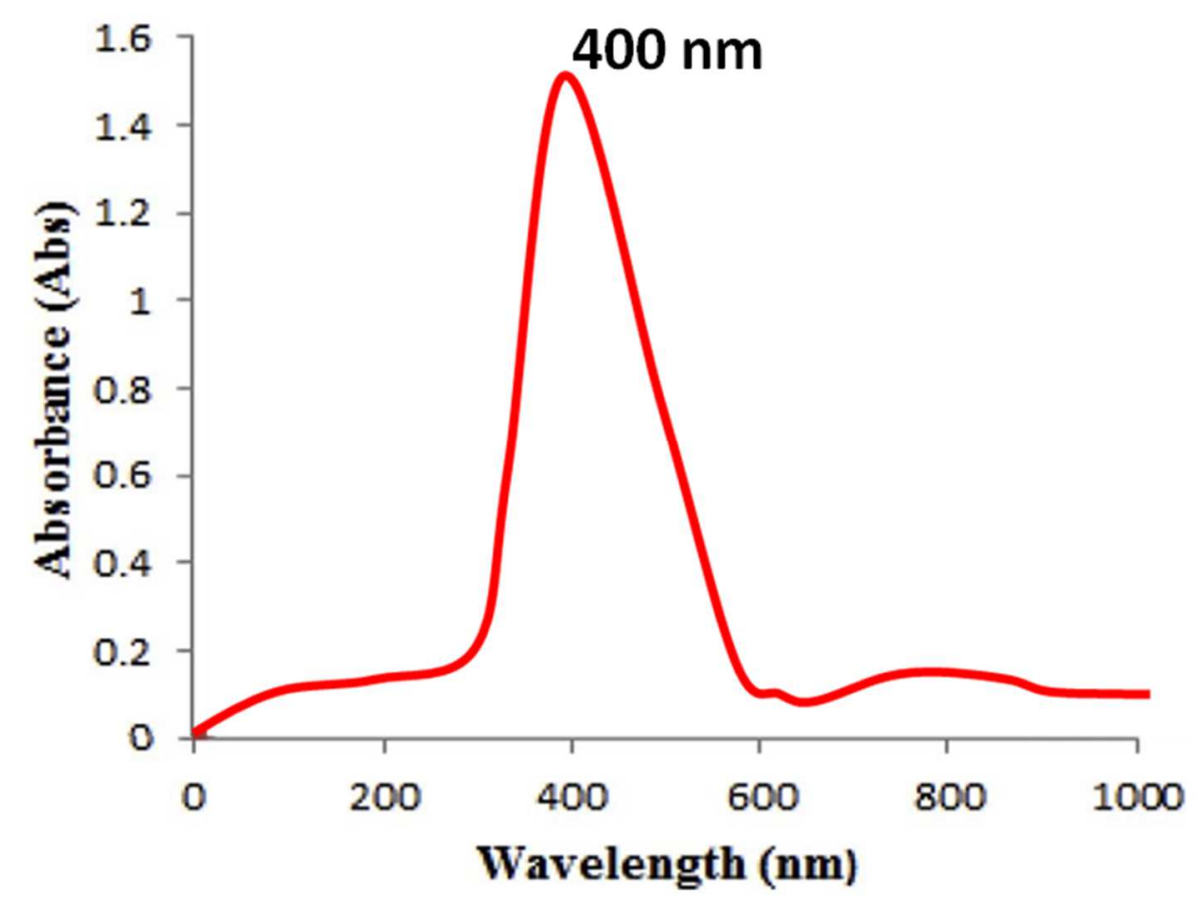

Fig 1. UV-Vis absorption spectrum of $\mathrm{TiO}_{2} \mathrm{NPs}$ prepared using Lippia citriodora extract

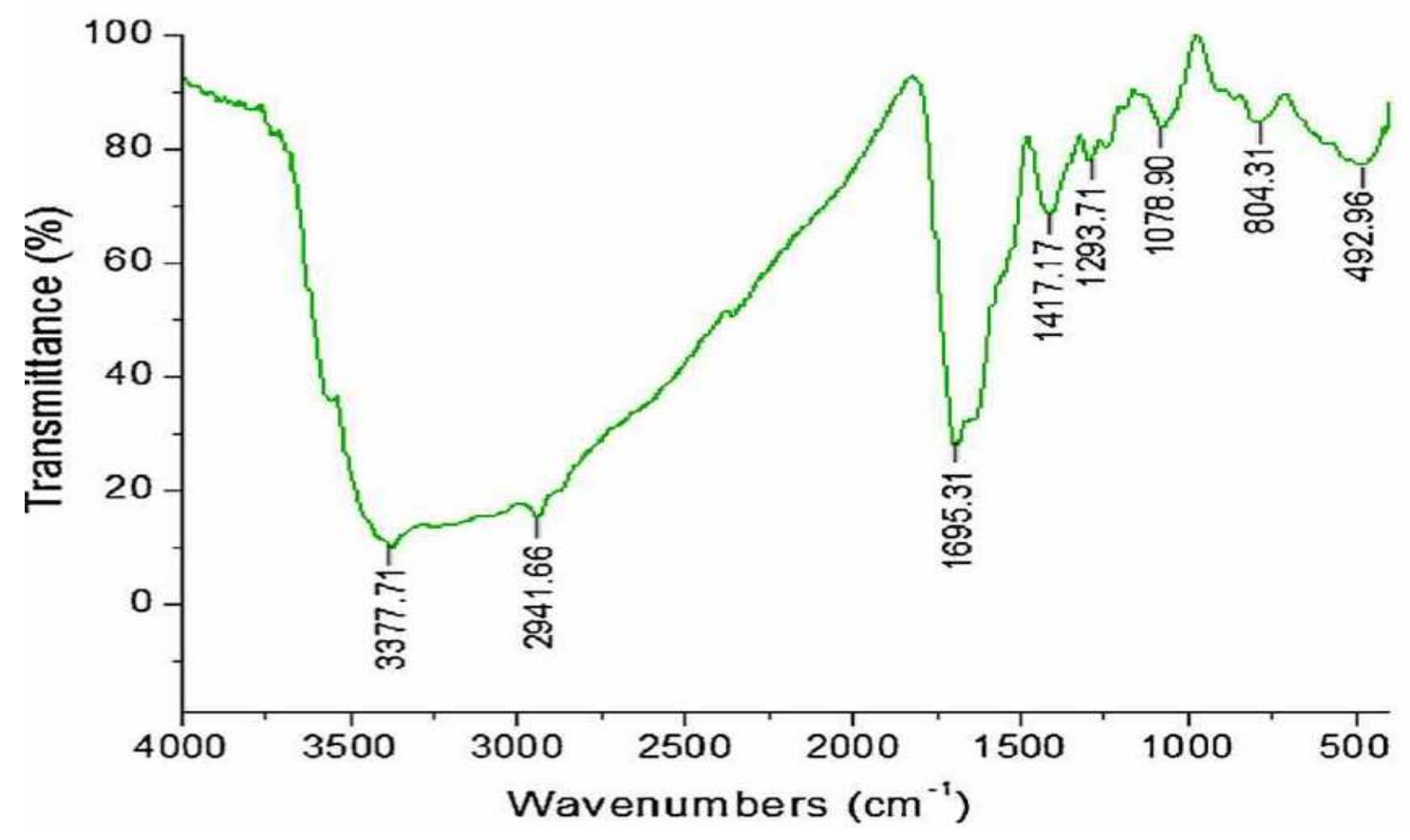

Fig.2. FTIR peaks of $\mathrm{TiO}_{2}$ NPs. 
The XRD diffraction was used for investigating the structural phase of $\mathrm{TiO}_{2}$ nanoparticles as indicated in Fig. 3. The XRD structure shows that the powder of $\mathrm{TiO}_{2}$ constitutes of mixed rutile and anatase phases which belongs to the space groups $\mathrm{P} 4_{2} / \mathrm{mnm}$ with $\mathrm{Z}$ value as 2 and $\mathrm{I} 4_{1} /$ amd with $\mathrm{Z}$ values as 4 , accordingly. The phase fraction in the form of weight was observed to be $14.9 \%$ of rutile and $85.1 \%$ of anatase according to the method of Rietveld Refinement executed in software Match-2. The XRD structure of green $\mathrm{TiO}_{2}$ reveals great crystallinity exhibiting strong bands at $2 \theta$ values and the assigned anatase titania planes are $25.28(101), 37.81(004), 48.04(200), 54.22(105), 55.16(211), 62.73(204)$, 69.01(116), 70.18 (220), and 74.97(215), accordingly [9] (JCPDS 83-2243). Alternatively, the distinctive diffraction bands at $2 \theta$ values and the assigned rutile titania crystal phase are 27.42(110), 36.11(101), 41.28(111) and 55.06(220), accordingly [9] (JCPDS 77-0441). The XRD structure is utilized to analyse the crystal size for rutile $(\sim 24 \mathrm{~nm})$ and anatase phase $(\sim 13 \mathrm{~nm})$ with the help of Scherrer's equation.

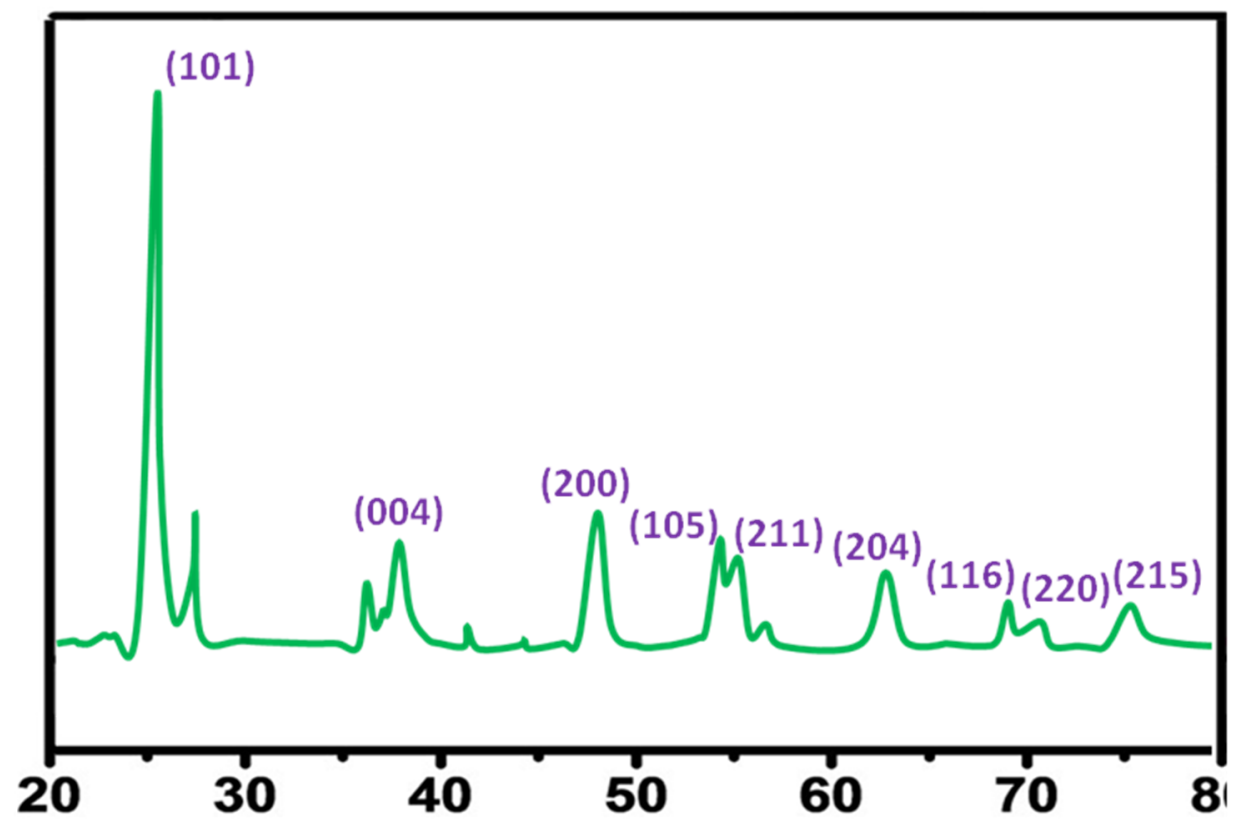

Fig.3. XRD pattern of $\mathrm{TiO}_{2} \mathrm{NPs}$ synthesized using plant extract 
For confirming the phase of green $\mathrm{TiO}_{2}$ material, raman spectroscopy was employed as represented in Fig. 4. The Raman spectroscopy displays distinctive peaks cantered at 637 $\left(\mathrm{E}_{\mathrm{g}}\right), 514\left(\mathrm{~A}_{1 \mathrm{~g}}\right), 397\left(\mathrm{~B}_{1 \mathrm{~g}}\right), 196\left(\mathrm{E}_{\mathrm{g}}\right)$ and $143 \mathrm{~cm}^{-1}\left(\mathrm{E}_{\mathrm{g}}\right)$ illuminating anatase phase [10], whereas the other fragile peak situated at $637 \mathrm{~cm}^{-1}\left(\mathrm{~A}_{1 \mathrm{~g}}\right)$ and $446 \mathrm{~cm}^{-1}\left(\mathrm{E}_{\mathrm{g}}\right)$ could be recognized as attribute of the rutile phase [11]. In green $\mathrm{TiO}_{2}$, the peak intensity clearly shows that the rutile phase percentage is less than the anatase phase percentage.
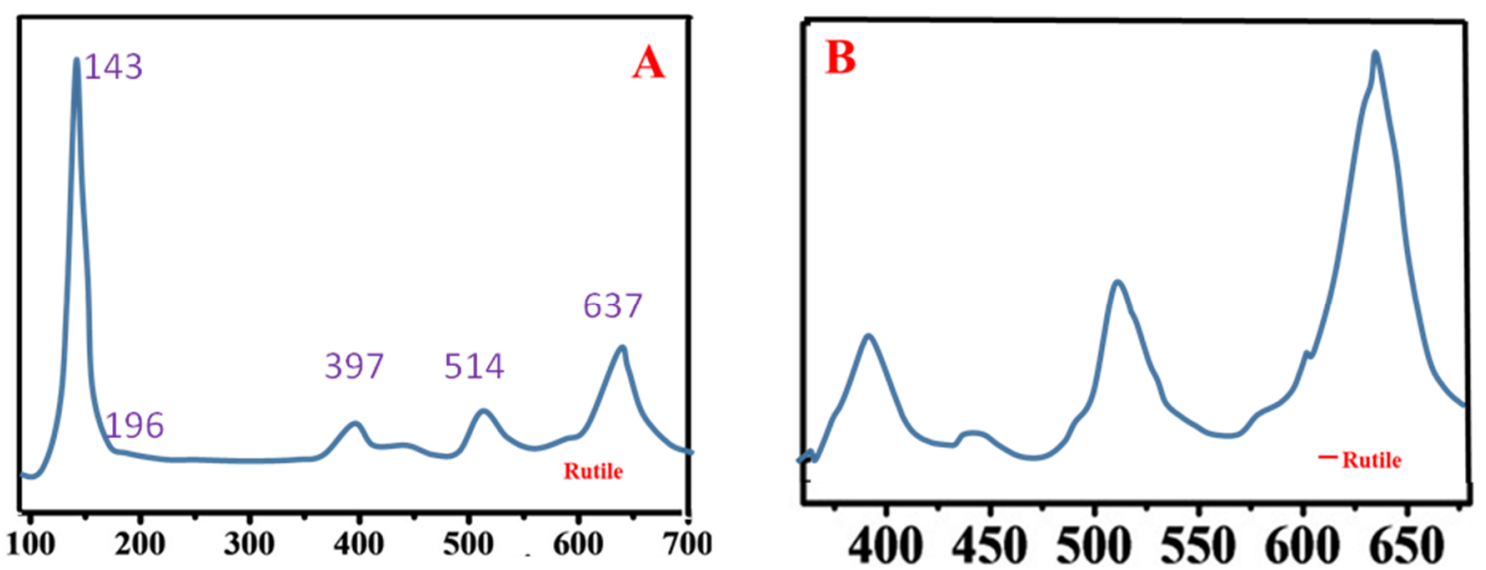

Fig. 4. Raman spectrum of rutile $\mathrm{TiO}_{2} \mathrm{NPs}$ synthesized using plant extract (A,B)

Fig.5A. showed the aggregates of close spherical NPs of 20-40 nm. There is constant distribution of elements. The crystalline particle formation is indicated by the TEM image (Fig. 5B). The size of the particle noticed from the TEM image is in 20-40 nm range. Also, EDS spectrum shown in Fig.6. represented the presence of elemental peaks corresponding to Titanium and oxygen confirmed the formation of $\mathrm{TiO}_{2} \mathrm{NPs}$ using plant extract. 

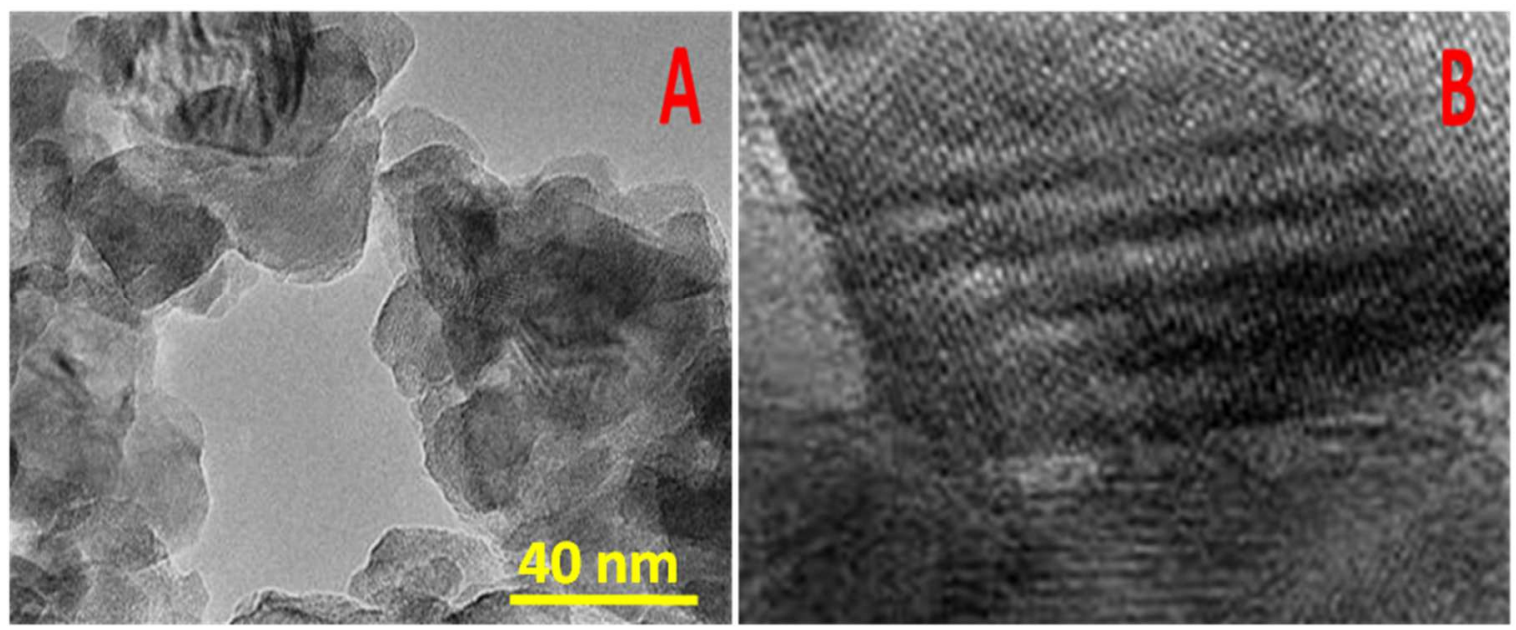

Fig.5. TEM results of $\mathrm{TiO}_{2} \mathrm{NPs}$ at high (A) and low magnification (B)

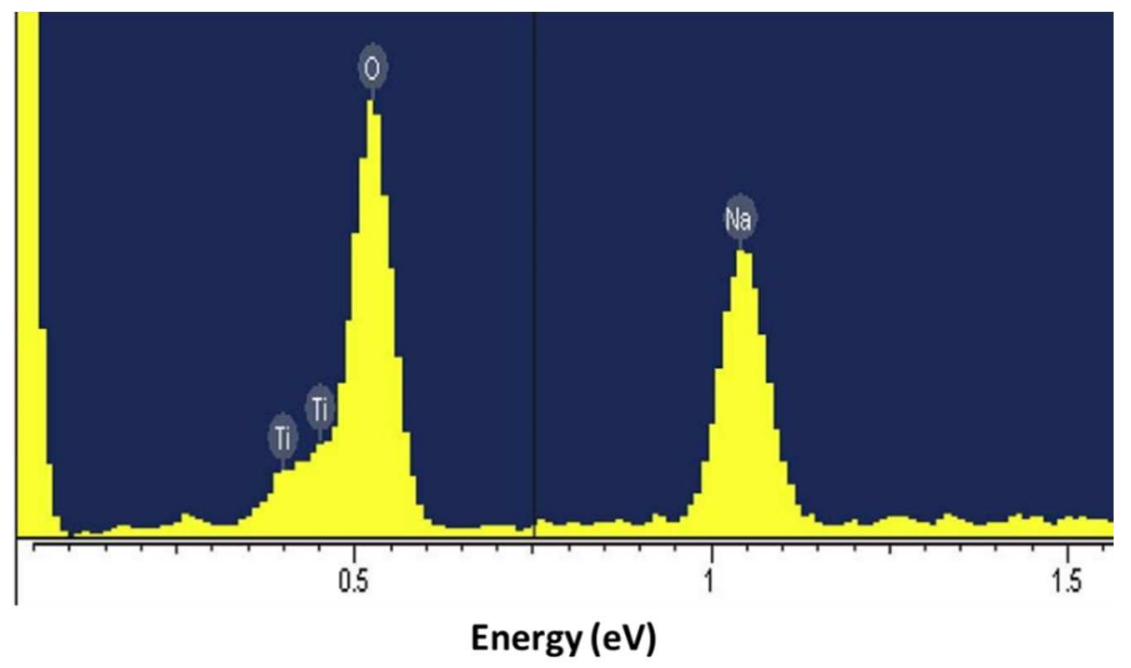

Fig.6. EDS spectrum fabricated $\mathrm{TiO}_{2} \mathrm{NPs}$

In vitro studies experiments

Principal cortical cultures of mouse embryos were revealed to various dosages of $\mathrm{TiO}_{2} \mathrm{NPs}(20,15,10,5,0 \mu \mathrm{g} / \mathrm{ml})$ for time intervals ranging 96 to $6 \mathrm{~h}$ to assess the impact of these nanoparticles on microglia, astrocytes, neurons and cell propagation. Various studies 


\title{
Equitable location of facilities in a region with probabilistic barriers to travel
}

\begin{abstract}
This paper studies a planar multi-facility location problem that considers the presence of a restricted region with probabilistic position. This problem seeks to locate facilities in an equitable manner by minimizing the maximum expected distance traveled from demand points to access a facility, as well as distances between locations of new facilities. We propose a heuristic to solve this problem that combines a bounding approach with a split-divide-and-conquer strategy. Computational study shows that this heuristic produces high-quality solutions in reasonable run-times. We report findings from a case-study involving locating police facilities in Kingston-Upon-Thames.
\end{abstract}

Keywords: facility location; fairness criterion; probabilistic line barrier; relaxation-based heuristic

\section{Introduction}

The problem of locating facilities to enable the efficient provision of services or products to individuals is a classical and long-studied problem. Facilities that serve a common or public good (such as an ambulance or a police station) and are to be located in an urban setting bring additional complexities to this location problem (Heizer and Render, 2011). In this context, achieving an appropriate response time (or distance) while considering fairness is a crucial factor for emergency service providers (Zhang et al., 2016). For example, a decision maker may face the challenges of siting a set of emergency service centers such that each person experience equitable distribution of response times. From a practical point of view, the effect of emergency facility location is dependent to its distance to the people utilizing it.

In many supply chain and logistics settings it is common to use an objective that seeks to minimize the sum of all distances traveled to/from facilities and individual demand sites. However, for locating emergency-related facilities (fire, ambulance, and police stations, etc.) such an objective is often inappropriate, as equity is often a consideration in such decisions. As such, an objective that rewards an equitable location of facilities with respect to individual demand sites is often used.

A variety of metrics for addressing equity, especially in the public sector, has been studied in the literature (Marsh and Schilling, 1994). We applied the maximum distance metric as an explicit consideration of fairness or equitability for the sake of computational tractability and managerial appropriateness. This measure is first used in Hakimi (1965). One such measure is to minimize not the sum of all distances but the maximum distance from a facility to an individual demand site. We use such an objective (sometimes called a minimax objective) in the research presented in this paper to address the equitable access to public emergency services (Marsh and Schilling, 1994). This objective has long been considered as a more equitable alternative comparing to the minisum problem which minimizes total travelled distance, since the minimax objective seeks to improve as much as possible providing services to those who are farthest from a service center, (Richard et al. 1990). More precisely, the minimax objective minimizes the maximum travelled distance from the nearset facilities to demand 
points. Equity can be regarded from different perspectives and therefore particular definition and perception may be used for each perspective. For example, in emergency context, equity from patient's perspective is totally different from the emergency service provider. A complete range equity definitions and formulations are proposed in McLay and Mayorga, (2013) and Bélanger et al., (2019).

Locational decisions should also recognize geographical or physical restrictions to travel. Such contexts could be studied under the discrete location framework, by defining the appropriate location-routing problem (Laporte and Dejax, 1989), location-transportation problem (Klibi et al., 2010), urban freight transportation problem (Marcucci and Gatta, 2014), or service network design problem (Lium et al., 2009) depending on the type of freight, the decisional level and the stakeholders involved. However, in several real-life cases, the approximations and aggregations employed in these models to capture the essence of the problem have not considered realistic geographical or physical restrictions. In such situations, predetermined allocated areas to each facility may no longer be the optimal one. For instance, while reaching affected areas in the shortest possible distance (or time) is crucial, the presence of barriers (i.e., obstructions) in city areas can affect the relief services. In an urban area, barriers may have probabilistic nature, with random presence, random size, random shape, or random location. Thus, the existence of barriers and their stochastic nature, should be taken into account when modeling the location problem.

Turning to locating facilities in urban settings, many (if not most) location models assume movements between located facilities or a facility and an individual's site are unrestricted. However, in many settings such an assumption is not realistic; instead an organization must locate facilities in a region that contains restricted areas. Such restricted areas can be placed into one of three categories: (1) forbidden regions (Batta et al. 1989), (2) congested regions (Sarkar et al. 2007), and (3) barrier regions (Hamacher and Nickel 1998). A forbidden region is one where a facility cannot be located but moving through is permitted (e.g. college campus, downtowns in large urban areas, etc.). A congested region is like a forbidden region, in that a facility may not be located in such a region but moving through is permitted. However, a congested region is different in that movement through such a region comes at additional cost or difficulty (e.g. tropical and subtropical deserts, forests, lakes, etc.). Finally, a barrier region is one where a facility cannot be located and movement through is not permitted, except for at some pre-defined passages. The impact of the presence of barrier in urban service facility location problems, in both practice and theory, has been extensively studied in Zanjirani Farahani et al., (2019). Our results also emphasize the fact that considering barriers has significant impact on the location of facilities in urban areas and that ignoring them may mislead the urban planners and city logistic managers.

When locating facilities in areas that contain barrier regions, point-to-point distances are inaccurate, as the barrier can impact both transportation times and distances. Thus, methods that locate facilities based on point-to-point distance measurements are likely to yield sub-optimal locations. Instead, one should use a distance function that measures the distance from facilities to individual demand sites while recognizing the location and size of the barrier. Such functions are often based on the calculation of shortest paths between individual demand sites and facilities in the presence of a barrier. In problems where multiple facilities must be located and close proximity between them is also desired, such functions should also be used to measure the distance between pairs of facilities.

In this paper, we present a method for locating multiple facilities when barrier regions are present and, as 
the facilities are located in an urban area wherein streets often form a grid, distance is measured on a rectilinear (Manhattan) basis. As we will see in the next section, the academic literature on location problems wherein barrier regions are present can be categorized by the nature of the barrier (e.g. polyhedral-shaped, arbitrarilyshaped, linear, etc.) as well as by whether the barrier has a fixed location that is known a priori. We propose a method for when the barrier is linear, and its presence and location are not known a priori and with certainty (i.e. a probabilistic line barrier).

At the heart of this method is a mixed integer nonlinear program that seeks to minimize the expected longest distance traveled, either from a facility to an individual demand site or between facilities. Considering the expected distance between facilities brings additional complexity to the problem we seek to solve. Many of the related problems that only consider distances from facilities to demand sites can be modeled as a convex optimization problem. Problems of this class are easier to solve, computationally-speaking, than general nonlinear programs, in part because they are known to have a globally optimal solution and there are tests for assessing whether a solution is optimal. The optimization problem we seek to solve, that consider both the distance between facilities and from facilities to individuals, has an objective and constraints that can not be guaranteed to be convex.

As such, we propose a heuristic algorithm that combines a bounding approach with a split-divide-andconquer strategy that can produce high-quality solutions to real-sized instances of the optimization problem in reasonable run-times. The bounding approach is based on a new type of relaxation for this type of problem, one based on presuming the barrier is fixed at its expected position. We analytically show that assuming the barrier is fixed at its expected position yields a relaxation that can be formulated as a mixed integer linear program. We computationally show that this relaxation is very tight, as solving it yields efficient lower bounds that are very close to the objective function value of known high-quality solutions.

While the model presented is relevant to many urban environments, in which accidents or construction (or reconstruction) projects, as inevitable events, may obstruct part of a road network and consequently cause interruption (detours and delays) in transportation distance or time (Sayarshad and Chow, 2017), and facility types (ambulances, fire stations, etc.), it has not yet been studied in the literature. Specifically, locating multiple facilities in the presence of a probabilistic barrier with the use of a minimax objective has not yet been addressed. Thus we believe that the model, as well as the computationally effective solution technique described in this paper, present two major contributions to the literature on facility location problems. As a final contribution, we illustrate the use of this method in practice with a case study based on locating police stations in Kingston Upon Thames. The method we propose is relevant to this specific problem as there is an overground rail line that runs through this area, which we model as a probabilistic line barrier. We analyze solutions produced by our method, which recognizes the barrier, as well as a method that does not, in order to assess how recognizing the barrier impacts the location of facilities and the expected longest distance travelled. We observe that recognizing the potential presence of this rail line can significantly reduce the expected longest distance traveled.

The reminder of the paper is organized as follows: The next section describes the academic literature relevant to this problem. Section 3 describes how the problem is modelled, whereas Section 4 presents our solution procedure. Section 5 then studies computationally the effectiveness of the solution procedure proposed 
and provides for an illustrative case the insights from the solution produced. Finally, Section 6 brings the paper to a conclusion, highlighting the strengths and weaknesses of the research presented and suggesting future research topics.

\section{Literature review}

A recent survey of the emergency facility location literature can be found in Bélanger et al., (2019) in which, minimax objective function, as the most often used equity measures in the emergency facility location problems, have been exhaustively studied. Zanjirani Farahani et al., (2019) has also reviewed service facility location problems in urban area and highlighted the importance of utilizing the minimax objective function in the emergency context to achieve equitability. As such, we focus our literature review on papers relevant to the problem we study, which is an equitable facility location problem with a barrier region in the continuous space. We first consider those that optimize a minisum objective and then those that optimize a minimax objective. We finish the section with a discussion of the contributions of this paper.

This paragraph reviews papers that optimize a minisum objective location problem with a fixed barrier. For the first time, Larson and Sadiq (1983) followed by Batta et al. (1989) studied the discretization properties of the planar Weber problem with the rectilinear distance function in the presence of polyhedral shaped barriers and showed that this problem can be reformulated as a $p$-median problem. Applying the same approach, Aneja and Parlar (1994) studied an Euclidean Weber problem with barrier regions to construct a visibility graph and to evaluate the shortest path between any candidate point for new facility and demand points using the simulated annealing (SA) algorithm. Hamacher and Klamroth (2000) developed a similar discretization for a general class of distance functions. Klamroth (2001a) proposed a reduction methodology for the same problem, in which the non-convex barrier location problem reduced to a set of convex location problems. Then, they presented an exact and a heuristic algorithm to solve the location problem with barriers. Klamroth (2001b) considered Weber location problems in the presence of a line barrier with a finite number of passages and presented a solution algorithm with exponential time complexity with respect to the number of passages. Klamroth and Wiecek (2002) proposed an algorithm for multi-criteria location problems with a fixed position line barrier. Dearing and Segars $(2002 \mathrm{a}, \mathrm{b})$ extended a rectilinear distances facility location problem to a more general class of location problems, developed a decomposition approach on which the objective function of a location problem with barriers is convex, and then optimized the problem using convex optimization methods. Pfeiffer and Klamroth (2005) worked on a $p$-norm distance Weber problem in the continuous space and some intermediate points that combines the continuous location models and network location models. Considering such barriers, Bischoff et al. (2009) presented the Euclidean multi-facility location-allocation problem and proposed two heuristics to solve the problem. Relevant literature studied the Weber location problem with minisum objective function and used a solution space discretization approach to solve the problem, while we keep the solution space continuous as in- the original problem when proposing the solution approach.

In the presence of finite-sized facilities and polyhedral/arbitrary shaped barriers, Savaş et al. (2002) first considered a single finite-sized facility location problem with rectilinear distance metric. Wang et al., (2002) formulated a mathematical programming model with minisum objective function where facilities are finite-sized or point and barriers are rectangular. Kelachankuttu et al. (2007) presented a single finite-sized 
facility location problem applying a contour line. Klamroth (2004) divided the feasible region into some convex regions, in which the number of these convex regions is bounded by $\mathrm{O}\left(N^{2}\right)$ where $N$ is the number of demand points. Bischoff and Klamroth (2007) solved that problem using the Weiszfeld technique and genetic algorithm (GA). These works considered the finite-size facility location problems in the presence of fixed position arbitrary shaped barriers. The related literature studied the Weber location problem in the presence of a fixed-position barrier. In this paper, we focus on a minimax location problem with a barrier which has a probabilistic position.

Miyagawa (2010) provided an analytical study on the rectilinear deviation distance from preplanned distance to visit a facility on a continuous plane with random pattern. Miyagawa $(2012,2017)$ studied the presence of a square and rectangular barrier in the probabilistic rectilinear distance Weber location problem, respectively, and showed that how the location and the size of the barrier affect the barrier distance and consequently the new facility location. Canbolat and Wesolowsky (2010) formulated a rectilinear distance single facility Weber location problem in the presence of a barrier, which randomly affects the distance between pairs of existingnew facilities; as such, an expected barrier distance function is computed. Proposing an exact algorithm, they proved that the expected barrier distance in each subspace is a convex function and that the Weber problem with a probabilistic line barrier is a convex optimization problem knowing that sum of a number of convex functions is a convex function. Considering this property, Shiripour et al. (2012) extended a multi-Weber location problem with a probabilistic line barrier and formulated a mixed integer quadratic programming model in the convex solution space. Amiri-Aref et al. (2013a) developed an exact algorithm to solve a generic model with a polyhedral-shaped barrier. Javadian et al. (2014) proposed a mixed-integer nonlinear programming (MINLP) model for the minisum location relocation problem with barrier and solved the problem in various sizes by implementing two meta-heuristics, GA and imperialist competitive algorithm (ICA). Oğuz et al. (2016) formulated a general mathematical model for facility location problems with restricted regions and Oğuz et al. (2018) proposed the Benders decomposition algorithm to solve the continuous location problem with restricted regions. While the existing research work mentioned above have shown the impact of randomness in the restricted location problem, the minimax objective function has not been studied in that context.

For the special case of minimax objective location problem, Nandikonda et al. (2003) considered the rectilinear distance location problem with arbitrary shaped barriers. Dearing et al. (2005) studied the minimax facility location problem with polyhedral barriers using the block norm distances and derived a finite dominating set for the problem. Then, Frieß et al. (2005) considered the minimax location problems in the presence of polyhedral barriers with the Euclidean distance. They proposed a solution approach based on propagation of circular wavefronts. Sarkar et al. (2007) extended that problem to a finite facility location problem with only new-exising interactions. These works considered the minimax location problems with fixed-position polyhedral barriers. Amiri-Aref et al. (2013b) took the advantage of the convexity of the expected barrier distance function in a multi-period planning horizon since the expected barrier distance was the summation of barrier distances over periods and as such, the summation function over period was convex. Considering that, they generated a convex multi-period rectilinear distance minimax location-dependent relocation problem that was sensitive to not only the demand but also the location of demand points during the planning horizon. Amiri-Aref et al. (2016) generalized the shape of barrier to polyhedral and proposed a threshold of the barrier size to be effective in the distance and a lower bound problem based on the forbidden region. We observe that all above-mentioned 
works consider the single facility minimax location problem, while we study a multi-facility minimax location problem in which the maximum distance between demand sites and facilities, as well as between facilities, is minimized. We note that while travel between facilities is common, this distance is usually not modelled in objective functions proposed in the literature.

We also summarize each reviewed article in this paper according to the general facility location problem classification, introduced by Hamacher and Nickel (1998), in Table 1, in which the first column outlines the related research works in the literature review, the second column represents the problem classification scheme, and the third column indicates the solution approach proposed for solving the corresponding problem. Notation used for problem classification scheme and solution approaches are listed at the bottom of Table 1. The classification is based on a five-position code as Pos $1 / \operatorname{Pos} 2 / \operatorname{Pos} 3 / \operatorname{Pos} 4 / \operatorname{Pos} 5$. Pos 1 indicates the number of new facilities that

Table 1: Literature review in facility location problems with barriers.

\begin{tabular}{|c|c|c|}
\hline Reference & Problem classification scheme* & Solution approach** \\
\hline \multicolumn{3}{|c|}{ minisum location problems with barriers } \\
\hline Larson and Sadiq (1983) & $1 / \Re^{2} / B=\mathrm{Ph} / d_{1}^{B} / \sum$ & Discretization heuristic \\
\hline Batta et al. (1989) & $1 / \Re^{2} / B=\mathrm{Ar} / d_{2}^{B} / \sum$ & Discretization heuristic \\
\hline Aneja and Parlar (1994) & $1 / \Re^{2} / B=\mathrm{Ph} / d_{2}^{B} / \sum$ & $\mathrm{DA}$ and $\mathrm{SA}$ \\
\hline Hamacher and Klamroth (2000) & $1 / \Re^{2} / B=\mathrm{Ph} / d_{p}^{B} / \sum$ & Discretization heuristic \\
\hline Klamroth (2001a) & $1 / \Re^{2} / B=\mathrm{Ph} / d_{2}^{B} / \sum$ & Reduction heuristic \\
\hline Klamroth (2001b) & $1 / \Re^{2} / B=\mathrm{Lp} / d_{2}^{B} / \sum$ & Decomposition approach \\
\hline Wang et al., (2002) & $1 / \Re^{2} / B=\operatorname{Re}, \mathrm{fs} / d_{1}^{B} / \sum$ & Heuristic \\
\hline Savaş et al. (2002) & $1 / \Re^{2} / B=\mathrm{Ph}, \mathrm{fs} / d_{1}^{B} / \sum$ & Heuristic \\
\hline Klamroth and Wiecek (2002) & $1 / \Re^{2} / B=\mathrm{Lp} / d_{L_{B}}^{B} / \sum$ & Decomposition approach \\
\hline Dearing and Segars (2002a) & $1 / \Re^{2} / B=\mathrm{Ph} / d_{1}^{B} / \sum$ & Decomposition approach \\
\hline Dearing and Segars (2002b) & $1 / \Re^{2} / B=\mathrm{Ph} / d_{1}^{B} / \sum$ & Partitioning heuristic \\
\hline McGarvey and Cavalier (2003) & $1 / \Re^{2} / B=\mathrm{Ph} / d_{2}^{B} / \sum$ & BSSS \\
\hline Klamroth (2004) & $1 / \Re^{2} / B=\mathrm{C} / d_{2}^{B} / \sum$ & Heuristic \\
\hline Pfeiffer and Klamroth (2005) & $N / \Re^{2} / B=\mathrm{Lp} / d_{L_{B}}^{B} / \sum_{p a r}$ & Discretization heuristic \\
\hline Bischoff and Klamroth (2007) & $1 / \Re^{2} / B=\mathrm{Ph} / d_{2}^{B} / \sum$ & Weiszfeld technique and GA \\
\hline Kelachankuttu et al. (2007) & $1 / \Re^{2} / B=\operatorname{Re}, \mathrm{fs} / d_{1}^{B} / \sum$ & Heuristic \\
\hline Bischoff et al. (2009) & $N / \Re^{2} / B=\mathrm{Ph} / d_{2}^{B} / \sum$ & Hybrid heuristic \\
\hline Miyagawa $(2010)$ & $1 / \Re^{2} / B=\operatorname{Re} / d_{1}^{B} / \sum_{\text {prob }} \vartheta_{\uparrow}$ & Analytical approach \\
\hline Canbolat and Wesolowsky (2010) & $1 / \Re^{2} / B=\mathrm{L} / d_{1}^{B} / \sum_{\text {prob }} \vartheta_{\uparrow}$ & Exact algorithm \\
\hline Shiripour et al. (2012) & $N / \Re^{2} / B=\mathrm{L} / d_{1}^{B} / \sum_{\text {prob }} \vartheta_{\uparrow}$ & ICA and GA \\
\hline Miyagawa (2012) & $1 / \Re^{2} / B=\mathrm{S} / d_{1}^{B} / \sum_{\text {prob }} \vartheta_{\uparrow}$ & Analytical approach \\
\hline Amiri-Aref et al. (2013a) & $1 / \Re^{2} / B=\mathrm{Ph} / d_{1}^{B} / \sum_{p r o b} \vartheta_{\uparrow}$ & Exact algorithm \\
\hline Javadian et al. (2014) & $1 / \Re^{2} / B=\mathrm{L} / d_{1}^{B} / \sum_{\text {prob }} \vartheta_{\uparrow}$ & ICA and GA \\
\hline Oğuz et al. (2016) & $N / \Re^{2} / B=\mathrm{Ar} / d_{p}^{B} / \sum \vartheta_{\uparrow}$ & CPLEX \\
\hline Miyagawa (2017) & $1 / \Re^{2} / B=\mathrm{Re} / d_{1}^{B} / \sum_{\text {par }} \vartheta_{\uparrow}$ & Analytical approach \\
\hline Oğuz et al. (2018) & $N / \Re^{2} / B=\mathrm{Ar} / d_{p}^{B} / \sum \vartheta_{\uparrow}$ & $\mathrm{BD}$ \\
\hline \multicolumn{3}{|c|}{ minimax location problems with barrier } \\
\hline Nandikonda et al. (2003) & $1 / \Re^{2} / B=\mathrm{Ar} / d_{1}^{B} / \max$ & Discretization heuristic \\
\hline Dearing et al. (2005) & $1 / \Re^{2} / B=\mathrm{Ph} / d_{2}^{B} / \sum$ or $\max$ & Decomposition approach \\
\hline Frieß et al. (2005) & $1 / \Re^{2} / B=\mathrm{Ph} / d_{2}^{B} / \max$ & Circular wave-front approach \\
\hline Sarkar et al. (2007) & $1 / \Re^{2} / B=\mathrm{Ar}, \mathrm{fs} / d_{1}^{B} / \max$ & Discretization heuristic \\
\hline Amiri-Aref et al. (2013b) & $1 / \Re^{2} / B=\mathrm{L}$, reloc $/ d_{1}^{B} / \max _{\text {prob }} \vartheta_{\uparrow}$ & ICA and GA \\
\hline Amiri-Aref et al. (2016) & $1 / \Re^{2} / B=\mathrm{L} / d_{1}^{B} / \max _{\text {prob }} \vartheta_{\uparrow}$ & Decomposition approach \\
\hline This paper & $N / \Re^{2} / B=\mathrm{L} / d_{1}^{B} / \max _{\text {prob }} \vartheta_{\uparrow} \vartheta_{\downarrow}$ & Split-divide-and-conquer algorithm \\
\hline \multicolumn{3}{|c|}{$\begin{array}{l}{ }^{*} 1 \text { : single facility, } N \text { :multi-facility, } \Re^{2} \text { :two-dimentional continuous space, } B \text { : barrier shape, C: circular, Ph: polyhedral, } \\
\text { Re: rectangular, S: square, Ar: arbitrary, L: line, Lp; line with passages, fs: finite size facility, par: Pareto locations, } \\
\text { prob: probabilistic, } \vartheta_{\uparrow} \text { : convex objective function } \vartheta_{\downarrow}: \text { concave objective function } \\
{ }^{* *} \text { BSSS: big square small square, DA: dijkstra's algorithm, GA: genetic algorithm, ICA: imperialist competitive } \\
\text { algorithm, SA: simulated annealing, BD: Benders decomposition, }\end{array}$} \\
\hline
\end{tabular}


should be located; Pos 2 shows the solution space that can be on the plane, discrete or network. Pos 3 refers to the special features of location problems (e.g., restricted region or relocation). Pos 4 displays the information about the interaction between the new facilities and the demand points such as distance or cost. Pos5 contains the objective function. The problem studied in this paper is represented by $N / \Re^{2} / B=\mathrm{L} / d_{1}^{B} / \max _{\text {prob }} \vartheta_{\uparrow} \vartheta_{\downarrow}$ stating that a planar multi-facility Weber location problem in a two-dimensional continuous space, i.e., $\Re^{2}$, is concerned. The special feature of this problem is the existence of a line-shaped barrier which has a probabilistic position on its route. The latter has been shown in Pos3 the problem classification scheme $(B=\mathrm{L})$. The rectilinear (Manhattan) distance function in the presence of the probabilistic line barrier is used in the problem formulation, shown in Pos 4 as $d_{1}^{B}$. The objective function is the minimization of the maximum expected barrier distance $\left(d_{1}^{B}\right)$ between any pairs of existing demand site and facilities as well as between the facility locations themselves, which is represented in the fifth position $\left(\max _{\text {prob }}\right)$. The convexity of the objective function cannot be determined, therefore both signs $\vartheta_{\uparrow}$ and $\vartheta_{\downarrow}$ are used.

Overall, this paper makes the following contributions. First, this paper models a problem that is of particular relevance to the location of emergency service facilities serving urban areas, yet has not been studied in the literature. While much of the literature has addressed minisum location problems, as underlined in Table 1, this paper integrates the notion of fairness by modeling a minimax location problem. Secondly, this paper is the first attempt to model a planar multi-facility minimax location problem that recognizes the presence of a line barrier with probabilistic location. The resulting model is both non-linear and non-convex, which makes it harder to solve than the relevant problems studied in the literature (Table 1). As a result, the third contribution of this paper is a new solution method for the proposed model, which employs a split-divide-conquer resolution strategy. The main feature of this solution method is a new relaxation for the problem, that computational experiments indicate is often able to produce a strong lower bound. These contributions are supported by a case study involving the location of police facilities in Kingston upon Thames. This case study illustrates the value in the proposed model and managerial insights that can be gained from its solutions.

\section{Problem modeling approach}

In this section, we first describe some of the constructs we use to formulate the problem we study; a facility location problem wherein the presence of a recurrent (and sometimes irregular) line barrier must be recognized and locations are measured by a function that evaluates the longest distance from a facility to an individual demand site. After reviewing these constructs, we present the formulation itself.

\subsection{Background}

We let $\mathcal{I}=\left\{P_{i} \in \Re^{2}: i=1, \ldots, I\right\}$ be a finite set of demand points where $I$ is the number of demand points and $\mathcal{J}=\left\{X_{j} \in \Re^{2}: j=1, \ldots, J\right\}$ be the finite set of new facilities that should be located ( $J$ is the number of new facilities). We let $P_{i}=\left(a_{i}, b_{i}\right)$ denote the coordinates of the demand point $i$ and $X_{j}=\left(x_{j}, y_{j}\right)$ denote the coordinates of the new facility $j$. Both are defined in a feasible region of the plane. Let $B$ be a set of nonempty barrier regions in $\Re^{2}$. As such, facilities may only be located and travel may only occur in $\mathcal{F}=\Re^{2} \backslash \int(B)$, where $\int(B)$ is the interior region of the barrier. We let $D_{p}(X, Y)$ denote the $p$-norm distance between $X, Y \in \mathcal{F}$,

which is calculated as $D_{p}(X, Y)=\sqrt[p]{\sum\left[|X-Y|^{p}\right]}$. We let $D_{p}^{B}\left(P_{i}, X_{j}\right)$ denote the $p$-norm barrier distance from $P_{i}$ to $X_{j}$ in the presence of barriers and call this the $p$-norm barrier distance. 


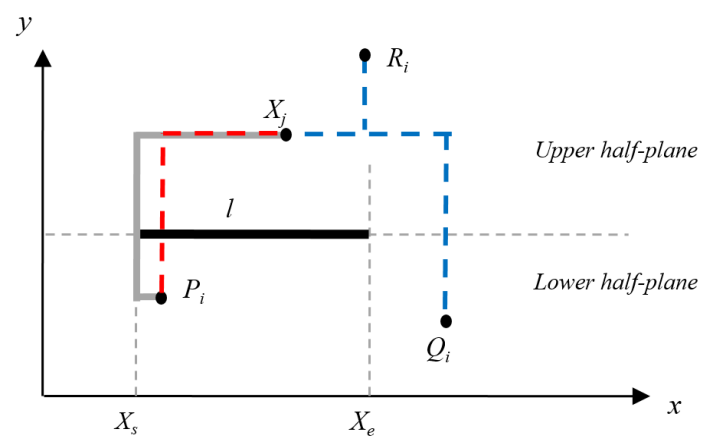

Figure 1: A line barrier.

We next use these distance functions to classify pairs of points based on the impact of the barrier on the distance between them. For points $X, Y \in \mathcal{F}$, we calculate $D_{p}^{B}(X, Y)=\inf \left\{l\left(\mathbf{P}_{X-Y}\right): \mathbf{P}_{X-Y}\right.$ is feasible $X-Y$ path $\}$, where $l\left(\mathbf{P}_{X-Y}\right)$ is the length of the feasible $X-Y$ path. We say the points $X$ and $Y \in \mathcal{F}$ are $p$-visible if $D_{p}^{B}(X, Y)=D_{p}(X, Y)$, i.e. the barrier does not impact the $p$-norm distance between them. For $X \in \mathcal{F}$ we define the set of visible points as visible $(X)=\left\{Y \in \mathcal{F}: D_{p}^{B}(X, Y)=D_{p}(X, Y)\right\}$. On the other hand, we say the points $X, Y \in \mathcal{F}$ are $p$-shadow if $D_{p}^{B}(X, Y)>D_{p}(X, Y)$, i.e. the barrier does affect the $p$-norm distance between them. For $X \in \mathcal{F}$ we define the set of shadow points as $\operatorname{Shadow}_{p}(X)=$ $\left\{Y \in F: D_{p}^{B}(X, Y)>D_{p}(X, Y)\right\}$. While we define these concepts generally, in this paper we focus on rectilinear distance metric (i.e., $p=1$ ).

We illustrate these concepts in Figure 1. There we illustrate a horizontal line barrier, $(\cdot, \beta) \in \Re^{2}$, with starting point $X_{s}$ and ending point $X_{e}$ (when the line barrier has a known length $l$, the ending point of the line barrier can be calculated as $\left.X_{s}+l\right)$. The dashed lines between pairs of points $\left(\left(X_{j}, R_{i}\right),\left(X_{j}, Q_{i}\right),\left(X_{j}, P_{i}\right)\right)$ represent feasible paths when the line barrier is not present. We see in Figure 1 that when the line barrier is fixed in this position it does not affect the distance between either $X_{j}$ and $Q_{i}$ or $X_{j}$ and $R_{i}$; hence, the points are 1-visible to each other and have regular rectilinear distances (indicated by blue dashed-lines). However, in this position the line barrier does affect the distance between points $X_{j}$ and $P_{i}$ (indicated by a red dashed-line). So those are shadow points. In this case the barrier distance, $D_{p}^{B}\left(X_{j}, P_{i}\right)>D_{p}\left(X_{j}, P_{i}\right)$, should be used, wherein $D_{p}^{B}\left(X_{j}, P_{i}\right)$ measures the length of the grey solid line. A more detailed description and discussion of line barriers can be found in Klamroth (2002).

In the problem we consider, we do not assume that the position of the line barrier is fixed and known $a$ priori. As a result, we use a probabilistic model of the starting point of the line barrier (and in turn the location of the end point is also random). Canbolat and Wesolowsky (2010) consider a similar situation, which they model with a continuous uniform distribution for the starting point of the line barrier, $X_{s} \sim U\left(L_{1}, L_{2}\right)$ with density function $f\left(X_{s}\right)=\frac{1}{L_{2}-L_{1}}, L_{1} \leq X_{s} \leq L_{2}$. As the length of the line is known, the end point of the line will also follow a continuous uniform distribution. We let $r$ represent the difference between upper and lower limits of $X_{s}$, i.e. $r=L_{2}-L_{1}$. The expected barrier distance between points $x, a_{i}$ can be calculated as follows 
(Canbolat and Wesolowsky 2010):

$$
E\left[D_{1}^{B}\left(x, a_{i}\right)\right]=\left\{\begin{array}{ll}
\frac{\left(l-\left|x-a_{i}\right|\right)^{2}}{2 r}+\left|x-a_{i}\right| ; & \left|x-a_{i}\right|<l \\
\left|x-a_{i}\right| ; & \left|x-a_{i}\right| \geq l
\end{array}\right\} \forall i
$$

\subsection{Formulation}

To formulate the model we assume $I$ predetermined demand points located at $\left(a_{i}, b_{i}\right) \in \Re^{2}, i=1, \ldots, I$ and $J$ new facilities whose location we represent with $\left(x_{j}, y_{j}\right) \in \Re^{2}, j=1, \ldots, J$. We assume a line barrier with length $l$ and a starting point that is randomly distributed on the horizontal route $(\cdot, \beta) \in \Re^{2}, \cdot \in\left[L_{1}, L_{2}\right]$. We weight the distances between facilities and demand points with the scalar $w_{i j}$. This weight can represent the number of customers or volume of demand in location $i$ that can be served by facility $j$. Similarly, our problem setting also involves travel between facilities and thus we assume a weight parameter, $v_{j k}$ for that distance as well.

We first describe the objective of the model. We focus on the location of emergency service facilities. In this context, equitable accessibility is an important criteria. One meausre of equitability is the furthest distance an individual would have to travel to reach the facility. In our model we choose to minimize this furthest distance using what is often referred to as a minimax objective.

Given the notation just described, the maximum weighted rectilinear barrier distance between new facility $j$ and demand point $i$ is calculated as:

$$
f_{1}\left(\left(x_{1}, y_{1}\right), \ldots,\left(x_{J}, y_{J}\right)\right)=\max _{1 \leq i \leq I, 1 \leq j \leq J}\left\{w_{i j} \cdot D_{1}^{B}\left(P_{i}, X_{j}\right)\right\}
$$

where $D_{1}^{B}\left(P_{i}, X_{j}\right)$ is the rectilinear barrier distance between new facility $j$ and demand point $i$. Similarly, the maximum weighted rectilinear barrier distance function between new facilities $j$ and $k$ is:

$$
f_{2}\left(\left(x_{1}, y_{1}\right), \ldots,\left(x_{J}, y_{J}\right)\right)=\max _{1 \leq j<k \leq J}\left\{v_{j k} \cdot D_{1}^{B}\left(X_{j}, X_{k}\right)\right\}
$$

In summary, the maximum rectilinear barrier distance among all demand points and new facilities is as follows:

$$
f\left(\left(x_{1}, y_{1}\right), \ldots,\left(x_{J}, y_{J}\right)\right)=\max \left\{\max _{1 \leq i \leq I, 1 \leq j \leq J}\left\{w_{i j} \cdot D_{1}^{B}\left(P_{i}, X_{j}\right)\right\}, \max _{1 \leq j<k \leq J}\left\{v_{j k} \cdot D_{1}^{B}\left(X_{j}, X_{k}\right)\right\}\right\}
$$

Because the location of the barrier is random we will optimize the expected barrier distance between any pairs of points based on the following calculation:

$$
E\left[f\left(\left(x_{1}, y_{1}\right), \ldots,\left(x_{J}, y_{J}\right)\right)\right]=\max \left\{\max _{1 \leq i \leq I, 1 \leq j \leq J}\left\{w_{i j} \cdot E\left[D_{1}^{B}\left(P_{i}, X_{j}\right)\right]\right\}, \max _{1 \leq j<k \leq J}\left\{v_{j k} \cdot E\left[D_{1}^{B}\left(X_{j}, X_{k}\right)\right]\right\}\right\}
$$

As the barrier moves along a horizontal route it does not impact the distance traveled along the $y$-axis. As such, our optimization problem seeks to

$$
\begin{aligned}
& \min _{\left(x_{j}, y_{j}\right) \in \Re^{2}, j \in \mathcal{J}}\left\{E\left[f\left(\left(x_{1}, y_{1}\right), \ldots,\left(x_{J}, y_{J}\right)\right)\right]=\right. \\
& \left.\quad \max \left\{\max _{1 \leq i \leq I, 1 \leq j \leq J}\left\{w_{i j} \cdot\left(E\left[D_{1}^{B}\left(a_{i}, x_{j}\right)\right]+\left|b_{i}-y_{j}\right|\right)\right\}, \max _{1 \leq j<k \leq J}\left\{v_{j k} \cdot\left(E\left[D_{1}^{B}\left(x_{j}, x_{k}\right)\right]+\left|y_{j}-y_{k}\right|\right)\right\}\right\}\right\}
\end{aligned}
$$

For the model to determine whether or not the barrier distance should be used to measure the distance between a demand point and a facility or between two facilities we define variables and constraints associated 
with each dimension in the plane $\left(\Re^{2}\right)$. Recalling that $P_{i}=\left(a_{i}, b_{i}\right)$ denote the coordinates of the demand point $i$ and $X_{j}=\left(x_{j}, y_{j}\right)$ denote the coordinates of the facility $j$ we next describe these two sets of variables and constraints.

\subsubsection{Visibility constraints}

These variables and constraints examine the $\mathrm{x}$-coordinate of the demand point (or facility) and a facility to determine whether the barrier may be in effect. Specifically, these constraints are based on the observation that when demand point $i$ and new facility $j$ are such that $\left|x_{j}-a_{i}\right|<l$, the barrier may affect the distance between the two points $P_{i}$ and $X_{j}$ (and thus $D_{1}^{B}\left(P_{i}, X_{j}\right)$ should be used). Because this set of constraints deals with the visible points properties described earlier, we hereafter called them "visibility constraints". To model this we define the variables $p_{i j} \in\{0,1\}$, which should satisfy constraints (7).

$$
p_{i j}=\left\{\begin{array}{ll}
1 ; & \left|x_{j}-a_{i}\right|<l \\
0 ; & \left|x_{j}-a_{i}\right| \geq l
\end{array}\right\}, \quad 1 \leq i \leq I, 1 \leq j \leq J
$$

Similarly, we define the variable $t_{j k}$ to represent when a barrier may impact the distance between the facilities $j$ and $k$; these variables should satisfy constraints (8).

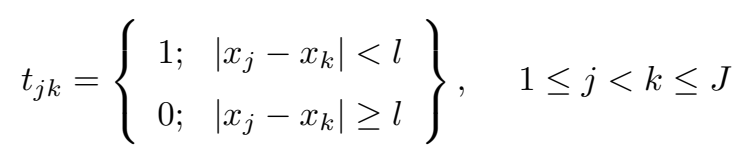

\subsubsection{No-flattening constraints}

The next set of variables and constraints examines the y-coordinates of a demand point and facility (or two facilities) to determine whether the barrier may be in effect. Given that the y-coordinate of the barrier is known, we divide the plane into two half-planes: 1) the half-plane under the barrier route and 2) the half-plane above the barrier route. When two arbitrary points are located in opposite half-planes, then the barrier may be in effect (see $X_{j}$ and $P_{i}$ in Figure 1), which is called "no-flattening condition". On the other hand, when two arbitrary points are in the same half-plane, the barrier does not affect the distance between them (see $X_{j}$ and $R_{i}$ in Figure 1). To model this, for each pair of demand point $i$ and facility location $j$, we define the variables $q_{i j} \in\{0,1\}$ which should satisfy constraints (9).

$$
q_{i j}=\left\{\begin{array}{c}
1 ; \quad\left(b_{i}>\beta \wedge y_{j}>\beta\right) \vee\left(b_{i} \leq \beta \wedge y_{j} \leq \beta\right) \\
0 ; \text { otherwise }
\end{array}\right\}, 1 \leq i \leq I, 1 \leq j \leq J
$$

As with the x-dimension, we also define the variable $u_{j k} \in\{0,1\}$ for the pair of facility locations $j$ and $k$. This variable should satisfy constraints (10).

$$
u_{j k}=\left\{\begin{array}{c}
1 ; \quad\left(y_{j}>\beta \wedge y_{k}>\beta\right) \vee\left(y_{j} \leq \beta \wedge y_{k} \leq \beta\right) \\
0 ; \text { otherwise }
\end{array}\right\}, \quad 1 \leq j<k \leq J
$$

\subsubsection{Barrier constraints}

The previous variables, appropriately defined and constrained, enable us to model when the barrier distance must be used to measure the distance between two points. To that effect, we define the binary variables $c_{i j}$ and $c_{j k}^{\prime}$ to represent when the barrier distance function should be used to measure the distance between demand 
point $i$ and facility $j$ or facilities $j$ and $k$. We relate these new variables to the dimension-specific variables with constraints (11) and (12).

$$
\begin{gathered}
c_{i j}=\left\{\begin{array}{cc}
1 ; & p_{i j}+q_{i j}=2 \\
0 ; & p_{i j}+q_{i j} \in\{0,1\}
\end{array}\right\}, 1 \leq i \leq I, 1 \leq j \leq J \\
c_{j k}^{\prime}=\left\{\begin{array}{cc}
1 ; & t_{j k}+u_{j k}=2 \\
0 ; & t_{j k}+u_{j k} \in\{0,1\}
\end{array}\right\}, \quad 1 \leq j<k \leq J,
\end{gathered}
$$

According to Canbolat and Wesolowsky (2010), the barrier conditions are only dependent on the xcoordinate and y-coordinate constraints aforementioned; however, Amiri-Aref et al. (2016) showed that the barrier condition is also sensitive to the length of the barrier and proposed a threshold of the length of barrier that may impact the barrier constraints. If the length of the barrier is long enough and the x-coordinate and y-coordinate constraints are met, the barrier distance will be greater than the regular distance, otherwise the regular rectilinear distance should be computed. Suppose $x$ and $x^{\prime}$ be the solution of the problem with barrier and without barrier, respectively. When the x-coordinate and y-coordinate conditions are true and the length of the barrier is long enough, the threshold of the length of barrier $l_{T}$ is defined as follows (for more information refer to Amiri-Aref et al. (2016)):

$$
l_{T}=\min _{i}\left\{\sqrt{2 r\left(\left|x^{\prime}-x_{i}\right|-\left|x-x_{i}\right|\right)}+\left|x-x_{i}\right|\right\}
$$

\subsubsection{Expected barrier distance computation}

Our model builds on the work presented in Canbolat and Wesolowsky (2010) wherein the expected value of the barrier distance is minimized when there is only one new facility. In particular, we expand their calculation to cases where there is more than one new facility. Constraints (14) state the conditions, given the starting point of the barrier $X_{s}$, when it impacts the distance between a facility $j$ and a demand point $i$ that are in different half-planes defined by the barrier route. We let $\varphi$ represent the value for $X_{s}$ that satisfy these conditions and $\rho\left(x_{j}\right)$ the probability that

$$
\rho\left(x_{j}\right)=\operatorname{Pr}\left(\max \left\{x_{j}-l, a_{i}-l\right\} \leq X_{s} \leq \min \left\{x_{j}, a_{i}\right\}\right), \quad 1 \leq i \leq I, 1 \leq j \leq J
$$

Considering the $\mathrm{x}$-coordinates of facility $j$ and demand points $i$ (again we presume they are in different half-planes defined by barrier route), there are two possible cases, (1) $x_{j}>a_{i}$ and (2) $x_{j} \leq a_{i}$. For the first case, the barrier distance should be used when $X_{s} \in\left(x_{j}-l,\left(x_{j}+a_{i}-l\right) / 2\right]$ and $X_{s} \in\left[\left(x_{j}+a_{i}-l\right) / 2, a_{i}\right)$. In the second, the barrier should be used when $X_{s} \in\left(a_{i}-l,\left(x_{j}+a_{i}-l\right) / 2\right]$ and $X_{s} \in\left[\left(x_{j}+a_{i}-l\right) / 2, x_{j}\right)$. As such, the expected barrier distance, conditioned on $X_{s} \in \varphi$ is given in equation (15).

$$
E\left[D_{1}^{B}\left(x_{j}, a_{i}\right) \mid X_{s} \in \varphi\right]=\frac{l+\left|x_{j}-a_{i}\right|}{2}, \quad 1 \leq i \leq I, 1 \leq j \leq J
$$

The other factor should be taken into account in the possibility of being in-effect of barrier occurrence on its route is its speed $\delta$. In fact the more speed the barrier has on its route, the less likely the barrier happens (i.e., $\left.\rho\left(x_{j}\right) \propto \frac{1}{\delta}\right)$. Therefore, the expected value of the distance between $x_{j}$ and $a_{i}$, when the barrier is in effect, is calculated in equation (16). 


$$
\begin{gathered}
E\left[D_{1}^{B}\left(x_{j}, a_{i}\right)\right]=\frac{l+\left|x_{j}-a_{i}\right|}{2} \rho\left(x_{j}\right)+\left|x_{j}-a_{i}\right|\left(1-\rho\left(x_{j}\right)\right) \\
=\frac{\left(l-\left|x_{j}-a_{i}\right|\right)^{2}}{2 r}+\left|x_{j}-a_{i}\right|, i \in I, j \in J
\end{gathered}
$$

The general expected barrier distance between $x_{j}$ and $a_{i}$ is presented in equation (17).

$$
E\left[D_{1}^{B}\left(x_{j}, a_{i}\right)\right]=\left\{\begin{array}{ll}
\frac{\left(l-\left|x_{j}-a_{i}\right|\right)^{2}}{2 r \delta}+\left|x_{j}-a_{i}\right| ; & \left|x_{j}-a_{i}\right|<l \\
\left|x_{j}-a_{i}\right| ; & \left|x_{j}-a_{i}\right| \geq l
\end{array}\right\}, \quad 1 \leq i \leq I, 1 \leq j \leq J
$$

Similarly, the expected barrier distance between pairs of the new facilities, i.e., $E\left[D_{1}^{B}\left(x_{j}, x_{k}\right)\right]$, can be computed in equation (18).

$$
E\left[D_{1}^{B}\left(x_{j}, x_{k}\right)\right]=\left\{\begin{array}{ll}
\frac{\left(l-\left|x_{j}-x_{k}\right|\right)^{2}}{2 r \delta}+\left|x_{j}-x_{k}\right| ; & \left|x_{j}-x_{k}\right|<l \\
\left|x_{j}-x_{k}\right| ; & \left|x_{j}-x_{k}\right| \geq l
\end{array}\right\}, \quad 1 \leq j<k \leq J
$$

To sum up, the minimization of the objective function (6) is reformulated to objective function (19) using the above mentioned equations:

$$
\min _{\left(x_{1}, y_{1}\right), \ldots,\left(x_{J}, y_{J}\right)}\left\{\max \left\{E\left[D_{1}^{B}\left(x_{j}, a_{i}\right)\right], E\left[D_{1}^{B}\left(x_{j}, x_{k}\right)\right]\right\}\right\}
$$

where:

$$
\begin{gathered}
E\left[D_{1}^{B}\left(x_{j}, a_{i}\right)\right]=\max _{\substack{1 \leq i \leq I \\
1 \leq j \leq J}}\left\{w_{i j} \cdot\left(\frac{\left(l-\left|x_{j}-a_{i}\right|\right)^{2}}{2 r \delta} \cdot c_{i j}+\left|x_{j}-a_{i}\right|+\left|y_{j}-b_{i}\right|\right)\right\} \\
E\left[D_{1}^{B}\left(x_{j}, x_{k}\right)\right]=\max _{1 \leq j<k \leq J}\left\{v_{j k} \cdot\left(\frac{\left(l-\left|x_{j}-x_{k}\right|\right)^{2}}{2 r \delta} \cdot c_{j k}^{\prime}+\left|x_{j}-x_{k}\right|+\left|y_{j}-y_{k}\right|\right)\right\}
\end{gathered}
$$

subject to constraints (7)-(12).

We let $z_{B}$ represents the objective function (19). We then write the proposed problem as below:

$$
\min _{\left(x_{1}, y_{1}\right), \ldots,\left(x_{J}, y_{J}\right)} z_{B}
$$

where

$$
\begin{array}{cc}
w_{i j} \cdot\left(\frac{\left(l-\left|x_{j}-a_{i}\right|\right)^{2}}{2 r \delta} \cdot c_{i j}+\left|x_{j}-a_{i}\right|+\left|y_{j}-b_{i}\right|\right) \leq z_{B}, & i \in I, j \in J \\
v_{j k} \cdot\left(\frac{\left(l-\left|x_{j}-x_{k}\right|\right)^{2}}{2 r \delta} \cdot c_{j k}^{\prime}+\left|x_{j}-x_{k}\right|+\left|y_{j}-y_{k}\right|\right) \leq z_{B}, & 1 \leq j<k \leq J
\end{array}
$$

subject to constraints (7)-(12).

\section{Solution approach}

As mentioned, this location problem with barrier is formulated as a nonlinear program, which can be difficult to solve, computationally-speaking. To overcome the computational challenge associated with solving this problem, we employ a solution method that first solves a relaxation of the problem wherein the barrier region is instead treated as forbidden. While a relaxation of the problem we seek to solve can be formed by simply ignoring the barrier region, a solution to this relaxation may locate a facility in such a region and thus be infeasible for the original problem. Instead, we formulate an optimization model wherein the barrier region is modeled 
as a forbidden region. As facilities are not allowed in forbidden regions, doing so implies that solutions to this model are feasible for the original problem. However, recall that traversing a forbidden region is allowed, while traversing a barrier region is not. Thus, distances in this model are underestimates of those incurred in the original problem. As a result, this model is a relaxation of the original problem. A formal proof that this model is a relaxation can be found in Klamroth (2002).After solving this relaxation the algorithm then evaluates the actual, expected, distances traveled from the locations it suggests when the probabilistic barrier is in place. If, in fact, the barrier does not impact these expected distances then the locations are optimal and the algorithm terminates. Conversely, if the barrier does impact the expected distances, the algorithm then proceeds to divide the multi-facility location problem into a series of single-facility location with a probabilistic barrier problems, where the solution space of each single-facility location problem is splitted into two subspaces. Finally, it conquers the best solutions from the subproblems. Figure 2 illustrates the algorithm.

In this section we first describe the steps taken by the algorithm in detail. We finish with a discussion of an alternate optimization problem we solve in order to benchmark the performance of the algorithm.

\subsection{Pre-processing step}

As stated before, establishing a facility in a forbidden region is prohibited but passing through is permitted. Accordingly, we present a relaxation of the multi-facility minimax location problem with a probabilistic line barrier based on considering a forbidden region along the barrier route. Klamroth (2002) first introduced this restricted location problem involving a forbidden region. Empirically-speaking, this has been known to yield a strong lower bound on the original problem. We present this lower bound problem below:

$$
z_{R}=\min _{\left(x_{1}, y_{1}\right), \ldots,\left(x_{J}, y_{J}\right)} z
$$

Subject to:

$$
\begin{aligned}
w_{i j} \cdot\left(\left|x_{j}-a_{i}\right|+\left|y_{j}-b_{i}\right|\right) \leq z, & i \in I, j \in J \\
v_{j k} \cdot\left(\left|x_{j}-x_{k}\right|+\left|y_{j}-y_{k}\right|\right) \leq z, & j, k \in J, j<k \\
x_{j}, y_{j}, z \geq 0, y_{j} \neq \beta, & j \in J
\end{aligned}
$$

As the barrier region is a line the likelihood of an optimal solution requiring a facility to be located in the barrier region is extremely low. As such, we treat the constraint $y_{j} \neq \beta$ heuristically by first solving the forbidden region problem without that constraint and then slightly perturbing the location of the facility if in fact it is located in the barrier region.

As the location problem with forbidden region is a relaxation, it under-estimates the barrier distances associated with the locations it prescribes. Thus, after solving the lower bound problem to get the location coordinates $X_{R}^{*}=\left\{\left(x_{j}^{*}, y_{j}^{*}\right), j=1, \ldots, J\right\}$, we solve the optimization problem given by the objective function (22) and constraints (7)-(12) and (23) and (24) with the location coordinate variables fixed to the coordinates indicated by $X_{R}^{*}$ (e.g. we fix $x_{j}=x_{j}^{*}, y_{j}=y_{j}^{*} \forall j \in J$ ). We label the objective function value of this problem $z_{B}\left(X_{R}^{*}\right)$. This latter objective function value represents the original problem evaluation with a non-optimal solution $X_{R}^{*}$, which can be considered as an upper bound value for the original problem.

As the forbidden region problem is a relaxation, when $z_{B}\left(X_{R}^{*}\right)=z_{R}$, we can conclude that the solution $X_{R}^{*}$ is in fact optimal for the problem with a probabilistic barrier and the algorithm can terminate. When instead 


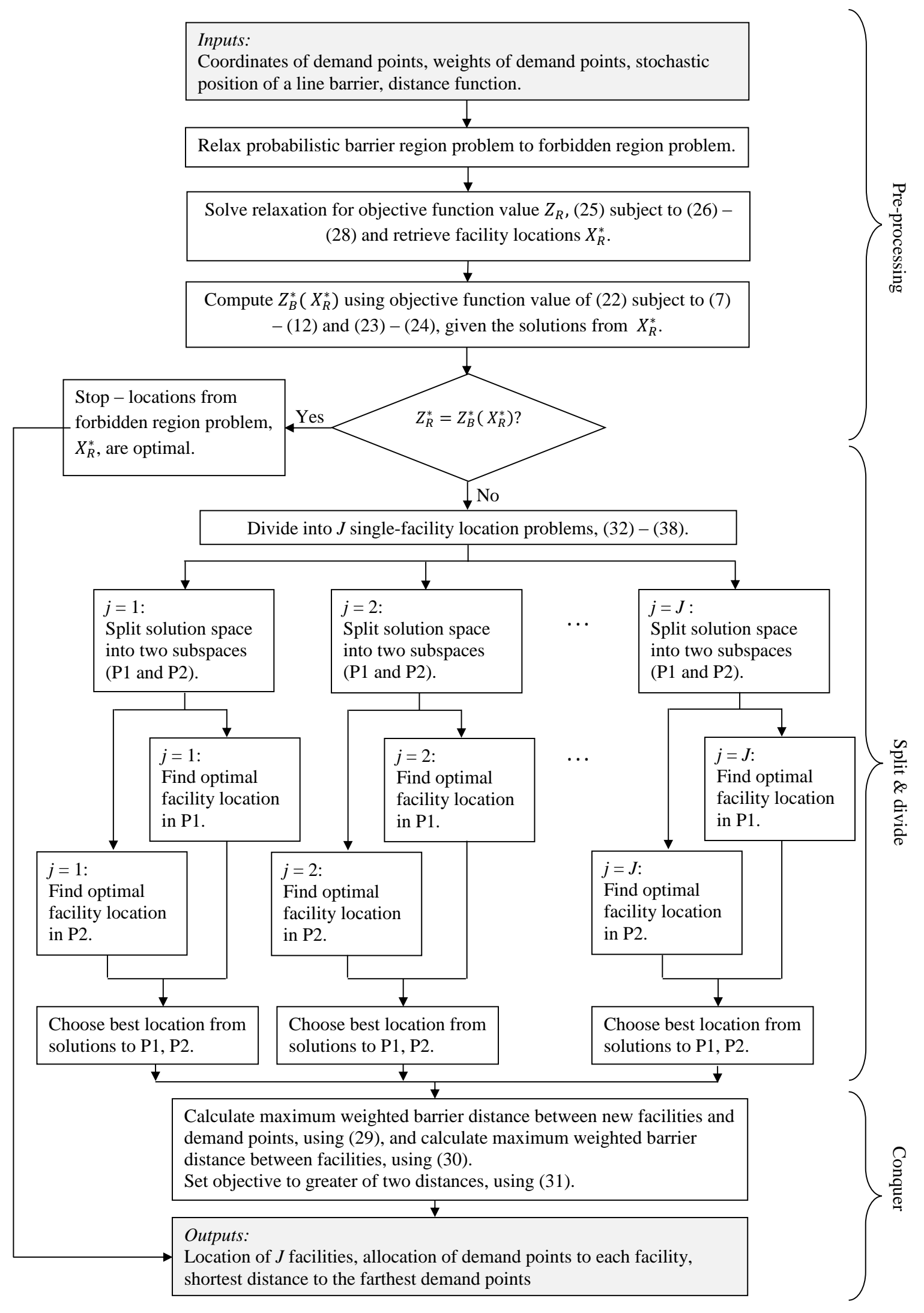

Figure 2: Flow chart of algorithm 
$z_{B}\left(X_{R}^{*}\right)>z_{R}$ the algorithm proceeds by decomposing the multiple-facility location problem into a series of single-facility location problems, as represented in Figure 2.

\subsection{Split-divide-and-conquer strategy}

We next discuss the split-divide-and-conquer portion of the algorithm. The proposed heuristic algorithm, which is based on a split-divide-and-conquer strategy, works in principle recursively and not iteratively, by breaking down the feasible set of the problem to optimize into several sub-problems of the main problem. It evaluates each subproblem to obtain efficient bounds on the subproblem objective value. The split-divide-and-conquer strategy with an implicit enumeration principle is a powerful tool for solving complex problems. For the considered minimax multi-facility location problem in the presence of a probablistic line barrier, this strategy first splits the whole solution space by the barrier route into two subspaces and then divides the original problem into a finite number of single-facility location sub-problems. The reason for which we adopted this heuristic algorithm is that the existing solution algorithms for solving the minimax multi-facility location problem with barrier are mostly based on the discretization approaches by applying continuous relaxation to discrete location problems. In contrast, the solution algorithm proposed in this paper maintains the continuity of the original problem and optimaly solves each sub-problem. This enables us to solve real-life sized problems.

The objective function (6) of the problem is composed of two terms: (1) the maximum expected barrier distance between facility locations and demand points, and, (2) the maximum expected barrier distance between facility locations. As such, for a given set of locations, one can calculate these two terms independently, with the objective function taking on the greater of the two.

In that spirit, when dividing the multi-facility location problem into a series of single-facility location problems, our algorithm solves a series of convex optimization problems that only consider the first term in the objective function (the expected barrier distance between the facility location and demand points). Then, after the location of all facilities has been determined, the maximum barrier distance between facilities is calculated. We define $z^{1}$ below to represent the maximum expected barrier distance between facilities and demand points, $z^{2}$ to represent the maximum expected barrier distance between facilities, and $z_{B}$ the greater of the two.

$$
\begin{gathered}
z^{1}=\min _{\left(x_{j}, y_{j}\right)}\left\{\max _{i, j}\left\{w_{i j} \cdot E\left[D_{1}^{B}\left(P_{i}, X_{j}\right)\right]\right\}\right\}, \text { subject to }(7),(9),(11), \text { and }(17) . \\
z^{2}=\min _{\left(x_{j}, y_{j}\right)}\left\{\max _{1 \leq j<k \leq J}\left\{v_{j k} \cdot E\left[D_{1}^{B}\left(X_{j}, X_{k}\right)\right]\right\}\right\} \\
z_{B}=\min \left\{z^{1}, z^{2}\right\}
\end{gathered}
$$

In this step, we divide the main optimization problem given by (32) into $J$ single-facility minimax location in the presence of a probabilistic line barrier problems. Specifically, we can formulate these single-facility location problems as (Further details regarding this reformulation can be found in A.1 in Appendix):

$$
z^{1}=\max _{j}\left\{z_{j}^{1}\right\} \quad \text { where } \quad z_{j}^{1}=\min _{\left(x_{j}, y_{j}\right)}\left\{\max _{i}\left\{w_{i j} \cdot E\left[D_{1}^{B}\left(P_{i}, X_{j}\right)\right]\right\}\right\}, \quad j \in J
$$

However, even solving this single-facility location problem may be computationally challenging due to the presence of a probabilistic line barrier. As such, we decompose this problem further, splitting the solution space 
into two subspaces; one is where the facility is located in the half-plane below the barrier region given by

- (35) (labeled P1 in the flow chart) and one is where the facility is located in the half-plane above the barrier region given by (36) - (38) (labeled P2 in the flow chart).

This strategy is based on the observation that the barrier will only impact the distance between a facility and the demand points in the opposite half-plane from where the facility is located. To make this discussion more precise, let $P_{i_{L}}=\left(a_{i_{L}}, b_{i_{L}}\right), i_{L}=1, \ldots, I_{L}$ be the coordinates of demand points in the lower halfplane and $P_{i_{U}}=\left(a_{i_{U}}, b_{i_{U}}\right), i_{U}=1, \ldots, I_{U}$ be the coordinates of demand points in the upper half-plane. Let $I_{L}=\left\{P_{i_{L}} \in \Re^{2} \mid b_{i_{L}}<\beta: i_{L}=1, \ldots, I_{L}\right\}$ be a finite set of demand points in the lower half-plane and let $I_{U}=\left\{P_{i_{U}} \in \Re^{2} \mid b_{i_{U}}>\beta: i_{U}=1, \ldots, I_{U}\right\}$ be a finite set of demand points in the upper half-plane.

To motivate splitting the solution space of this single-facility location problem into two subspaces, let $X_{j}^{*}=$ $\left(x_{j}^{*}, y_{j}^{*}\right)$ be the optimal location of $j$-th new facility. There are two possibilities for $X_{j}^{*}$, i.e., either $y_{j}^{*}<\beta$ (it is in the half-plane below the barrier) or $y_{j}^{*}>\beta$ (it is in the half-plane above the barrier). We next examine these two cases.

Case 1: when $y_{j}^{*}<\beta$, the actual distance between $X_{j}^{*}$ and $P_{i_{L}}$ is the regular rectilinear distance and the actual distance between $X_{j}^{*}$ and $P_{i_{U}}$ is the barrier distance. To model this case, we define problem P1 (for more details see A.2 in Appendix):

$$
\underline{z}_{j}^{1}=\max _{i_{L}, i_{U}}\left\{\underline{z}_{j i_{U}}^{1}, \underline{z}_{j i_{L}}^{1}\right\}, \quad j \in J \mid y_{j}^{*}<\beta
$$

where

$$
\begin{gathered}
\underline{z}_{j i_{U}}^{1}=\min _{\left(x_{j}, y_{j}\right)}\left\{\max _{i_{L}}\left\{w_{i_{L} j} \cdot\left(\left|a_{i_{L}}-x_{j}\right|+\left|b_{i_{L}}-y_{j}\right|\right)\right\}\right\}, j \in J, i_{L} \in I_{L} \\
\underline{z}_{j i_{L}}^{1}=\min _{\left(x_{j}, y_{j}\right)}\left\{\max _{i_{U}}\left\{w_{i_{U} j} \cdot\left(E\left[D_{1}^{B}\left(a_{i_{U}}, x_{j}\right)\right]+\left|b_{i_{U}}-y_{j}\right|\right)\right\}\right\}, j \in J, i_{U} \in I_{U}
\end{gathered}
$$

Case 2: Conversely, when $y_{j}^{*}>\beta$, the actual distance between $X_{j}^{*}$ and $P_{i_{U}}$ is the regular rectilinear distance and the actual distance between $X_{j}^{*}$ and $P_{i_{L}}$ is the barrier distance. To model this case, we define problem P2 (for more details see A.3 in Appendix):

$$
\bar{z}_{j}^{1}=\max _{i_{L}, i_{U}}\left\{\bar{z}_{j i_{L}}^{1}, \bar{z}_{j i_{U}}^{1}\right\}, \quad j \in J \mid y_{j}^{*}>\beta
$$

where

$$
\begin{gathered}
\bar{z}_{j i_{L}}^{1}=\min _{\left(x_{j}, y_{j}\right)}\left\{\max _{i_{U}}\left\{w_{i_{U} j} \cdot\left(\left|a_{i_{U}}-x_{j}\right|+\left|b_{i_{U}}-y_{j}\right|\right)\right\}\right\} \\
\bar{z}_{j i_{U}}^{1}=\min _{\left(x_{j}, y_{j}\right)}\left\{\max _{i_{L}}\left\{w_{i_{L} j} \cdot\left(E\left[D_{1}^{B}\left(a_{i_{L}}, x_{j}\right)\right]+\left|b_{i_{L}}-y_{j}\right|\right)\right\}\right\}
\end{gathered}
$$

We then calculate the location of facility $j \in J$ with $X_{j}^{*}=\operatorname{argmin}\left\{z_{j}^{1}\right\}$ Finally, the expected barrier distances between these facilities can be evaluated with the following:

$$
Z^{2}=\min \left\{\max _{1 \leq j<k \leq J}\left\{v_{j k} \cdot E\left[D_{1}^{B}\left(X_{j}^{*}, X_{k}^{*}\right)\right]\right\}\right\}
$$

We present a formal description of the algorithm below.

\section{Algorithm}

Inputs:

Coordinates of demand points and a probabilistic line-shaped barrier with known parameters 
1. Find $z_{R}^{*}$ and determine $X_{R}^{*}=\operatorname{argmin}\left\{z_{R}^{*}\right\}$

2. Evaluate $z_{B}\left(X_{R}^{*}\right)$

3. If $z_{R}^{*}=z_{B}\left(X_{R}^{*}\right)$ then

$$
X^{*}=X_{R}^{*}
$$

Go to End

Else

$$
\begin{aligned}
& \text { For } j=1 \text { to } J \\
& \qquad \begin{array}{r}
\underline{z}_{j}^{1}=\max \left\{\underline{z}_{j i_{L}}^{1} \underline{z}_{j i_{U}}^{1}\right\} \\
\bar{z}_{j}^{1}=\max \left\{\bar{z}_{j i_{L}}^{1}, \bar{z}_{j i_{U}}^{1}\right\} \\
z_{j}^{1}=\min \left\{\underline{z}_{j}^{1}, \bar{z}_{j}^{1}\right\} \\
X_{j}^{*}=\operatorname{argmin}\left\{z_{j}^{1}\right\}
\end{array}
\end{aligned}
$$

Next $j$

4. $\quad z^{1}=\max _{j}\left\{z_{j}^{1}\right\}$

$$
\begin{aligned}
& \text { For } j=1 \text { to } J-1 \\
& \qquad \begin{array}{l}
\text { For } k=j+1 \text { to } J \\
\text { Evaluate } z_{j k}^{2}\left(X_{j}^{*}, X_{k}^{*}\right)
\end{array}
\end{aligned}
$$

Next $j, k$

5. Find $z^{2}=\max _{j, k}\left\{z_{j k}^{2}\right\}$

6. $z_{B}=\max \left\{z^{1}, z^{2}\right\}$

End

\section{Outputs:}

Location of $J$ new facilities that minimize the maximum expected travel distance in the presence of a probabilistic line barrier.

The most time-consuming steps in the proposed algorithm are steps 4 and 5. Assume that runtime of each sub-problem in the algorithm is almost the same and equal $T$. Step 4 of the algorithm has time complexity of $O(4 J T)$, because four sub-problems should be solved to find $\underline{z}_{j i_{L}}^{1}, \underline{z}_{j i_{U}}^{1}, \bar{z}_{j i_{L}}^{1}$ and $\bar{z}_{j i_{U}}^{1}$ for each $j$. In step $5, z_{j k}^{2}$ should be evaluated $\frac{J(J-1)}{2}$ times; therefore, the time complexity of step 5 will be $O\left(\frac{J(J-1)}{2} T\right)$. To sum up, the time complexity of the algorithm in the worst case is $O\left(J^{2} T\right)$ where $T$ is the computational time for solving each sub-problem.

\subsection{Benchmark problem}

One of the challenges associated with solving the problem we study is the probabilistic nature of the location of the barrier. As such, to benchmark the computational performance of the proposed algorithm, we also compare it to the performance of solving a problem that removes this uncertainty by assuming the barrier is always located at its expected position. This is analogous to replacing a representation of the variability of a probability distribution with a point estimate that is its expected value. By assuming the line barrier is fixed at its expected position reduces the main problem to an optimization problem that is computationally easier to solve. To formulate this optimization problem we rely on the following two lemmas: 
Lemma 1. The expected value of start point of the line barrier is as follows:

$$
\bar{x}_{s}=\frac{L_{2}+L_{1}-l}{2}
$$

Proof. See the Appendix.

Lemma 2. Consider a line barrier at its expected position. When both visibility and no-flattening conditions are satisfied, the shortest path through two ends of the fixed line barrier computes the barrier distance.

Proof. See the Appendix.

With these lemmas and shortest paths in hand, we have the following optimization problem:

$$
z_{R P}=\min z^{\prime}
$$

Subject to:

$$
\begin{aligned}
& w_{i j} \cdot d_{i j}^{\prime} \leq z^{\prime} \\
& v_{j k} \cdot d_{j k}^{\prime} \leq z^{\prime} \\
& d_{i j}^{\prime}=\left\{\begin{array}{cc}
S P_{i j} & \bar{x}_{s} \leq x_{j} \leq \bar{x}_{e} \text { and } \bar{x}_{s} \leq a_{i} \leq \bar{x}_{e} \\
\left|a_{i}-x_{j}\right|+\left|b_{i}-y_{j}\right| & \text { otherwise }
\end{array} \quad i \in I, j \in J\right. \\
& d_{j k}^{\prime \prime}=\left\{\begin{array}{cc}
S P_{j k} & \bar{x}_{s} \leq x_{j} \leq \bar{x}_{e} \text { and } \bar{x}_{s} \leq x_{k} \leq \bar{x}_{e} \\
\left|x_{j}-x_{k}\right|+\left|y_{j}-y_{k}\right| & \text { otherwise }
\end{array} . \quad j, k \in J, j<k\right.
\end{aligned}
$$

where $\bar{x}_{e}, S P_{i j}$ and $S P_{j k}$ are formulated in equations (A.4), (A.5) and (A.6) in the Appendix, respectively. We further reformulate this nonlinear programming model to a mixed integer linear programming (MILP) model by linearization of the absolute value functions, multiplying binary variables, and multiplying binary to nonnegative variables. We note that this optimization problem, with the barrier fixed at its expected location, is also a reduction of the original problem wherein the location of the barrier is not known. This reduction consists of computing the distances $S P_{i j}$ and $S P_{j k}$ based on the expected location of barrier instead of the expected distance function.

\section{Computational results}

This section outlines the results of the computational performance of the algorithm. We study two dimensions of algorithm performance: (1) solution quality, and, (2) the time needed to produce a solution of that quality. We benchmark the algorithm on these metrics against three alternative methods: (1) solving the original problem with a non-linear solver (LINGO 14.0), (2) solving the MILP presented in Section 4.3 wherein the barrier is fixed at its expected location, and, (3) solving the relaxation problem wherein the barrier region is modeled as a forbidden region. The results consistently show that the algorithm proposed above is able to produce high-quality solutions in run-times that are comparable to what is necessary to solve the relaxation problems. We first discuss the setting and design for our experiments and then discuss the results. 


\subsection{Experimental design and setting}

All models and algorithms were implemented on a computer with 2 GB RAM and $2.00 \mathrm{GHz}$ processor running on Microsoft Windows 7. All non-linear programs (NLP) were solved with LINGO 14.0. Preliminary experiments indicated that the SLP (successive linear programming) directions strategy of LINGO was the best-performing and thus was used when solving NLP in this computational study. When solving NLPs the solver was given a time limit of three hours $(10,800$ seconds). If the optimal solution is not found at the end of the termination criterion, the software reports the best solutions found within the limit or the problem is considered non-solvable. The MILP presented in Section 4.3 was solved by CPLEX.

We randomly generated instances that have one of $5,10,20,50,100,200,500$ demand points for the computational study. For instances with no more than 20 demand points, we considered values from 2 to 5 facilities to open. For instances with at least 50 demand points, we tested values of 2, 4, 6 and 8 facilities to open. We also assume that starting point of the line barrier falls within a uniform distribution $U(0,12)$ and the $y$-coordinate of the line barrier is fixed at a horizontal barrier route, $\beta=8$. We also assume that the length of the line barrier is equal to 4 . In total, 26 sample problems were generated. All runtimes reported are in seconds.

\subsection{Results}

We present detailed results of our experiments, by instance, in Table 2. The table reports for each instance the objective function value $(\mathrm{OFV})$ of the solution along with the time required to produce that solution. The column labeled by "I" indicates the number of demand points in the instance and the column labeled by " $J$ " indicates the number of facilities to locate. We let $z_{B}$ and $t_{B}$ denote the objective function value and LINGO solver runtime, respectively, associated with the solution produced by solving the original problem with LINGO; $z_{B}^{A l g}$ and $t_{B}^{A l g}$ denote the objective function value and algorithm runtime, respectively, associated with the solution produced by the proposed algorithm; $z_{R}$ and $t_{R}$ denote the objective function value and solver runtime, respectively, associated with the solution produced by solving the relaxation that treats the barrier region as forbidden; and $z_{R P}$ and $z_{R P}$ denote the objective function value and CPLEX solver runtime, respectively, associated with the solution produced by solving the relaxation that treats the barrier as fixed at its expected position. For both the original problem and the relaxation that treats the barrier fixed at its expected position, LINGO may terminate with a solution that is only provably locally optimal. Columns $Z_{R}^{*}$ and $Z_{B}\left(X_{R}^{*}\right)$ indicate the objective function value of the optimal solution to the lower bound problem and the objective function value of the solution to the lower bound problem when evaluated by the real problem, respectively. Equality (or inequality) of these columns proves the globally (or locally) optimal solution of the results obtained from the lower bound problem. As such, in the column " $G / L$ " we indicate whether the algorithm is able to prove that the solution produced by solving the the problem with forbidden is globally optimal (indicated by " $G$ ") or locally optimal (indicated by " $L$ "). It is observed that for 14 of the 26 instances the algorithm was able to immediately converge to the optimal solution by solving the relaxation where the barrier region is treated as forbidden, before decomposing the problem into a series of single-facility location problems. For example, if we take the instance with $I=50$ and $J=2$, the equality of the objective function shows the globally optimal solution, although the location of new facilities obtained from each model is different. Regarding the solution produced by the proposed algorithm, column $z^{1}$ maximum barrier distance between new 
facilities and demand points and column $z^{2}$ represents the maximum barrier distance between facilities. To benchmark the performance of the algorithms we calculate three gaps:

1. Gap-exact: $\frac{z_{B}-z_{B}^{A l g}}{z_{B}}$ which measures the relative gap between the solution produced solving the original with LINGO and solution produced by the proposed algorithm. Note that LINGO can terminate at a locally optimal solution that is not globally optimal. As such, the proposed algorithm can (and in fact often does) produce a better solution.

2. Gap-fixed-barrier: $\frac{z_{B}-z_{R P}}{z_{B}}$ which measures the relative gap between the best solution produced by solving the original and solving the relaxation where the barrier is fixed at its expected position.

3. Gap-forbidden: $\frac{z_{B}-z_{R}}{z_{B}}$ which measures the relative gap between the solution produced by solving the original and solving the relaxation where the barrier region is modeled as a forbidden region.

Note that instances whose the relative gap is $0 \%$, indicating globally optimal solution, are represented in bold face. 


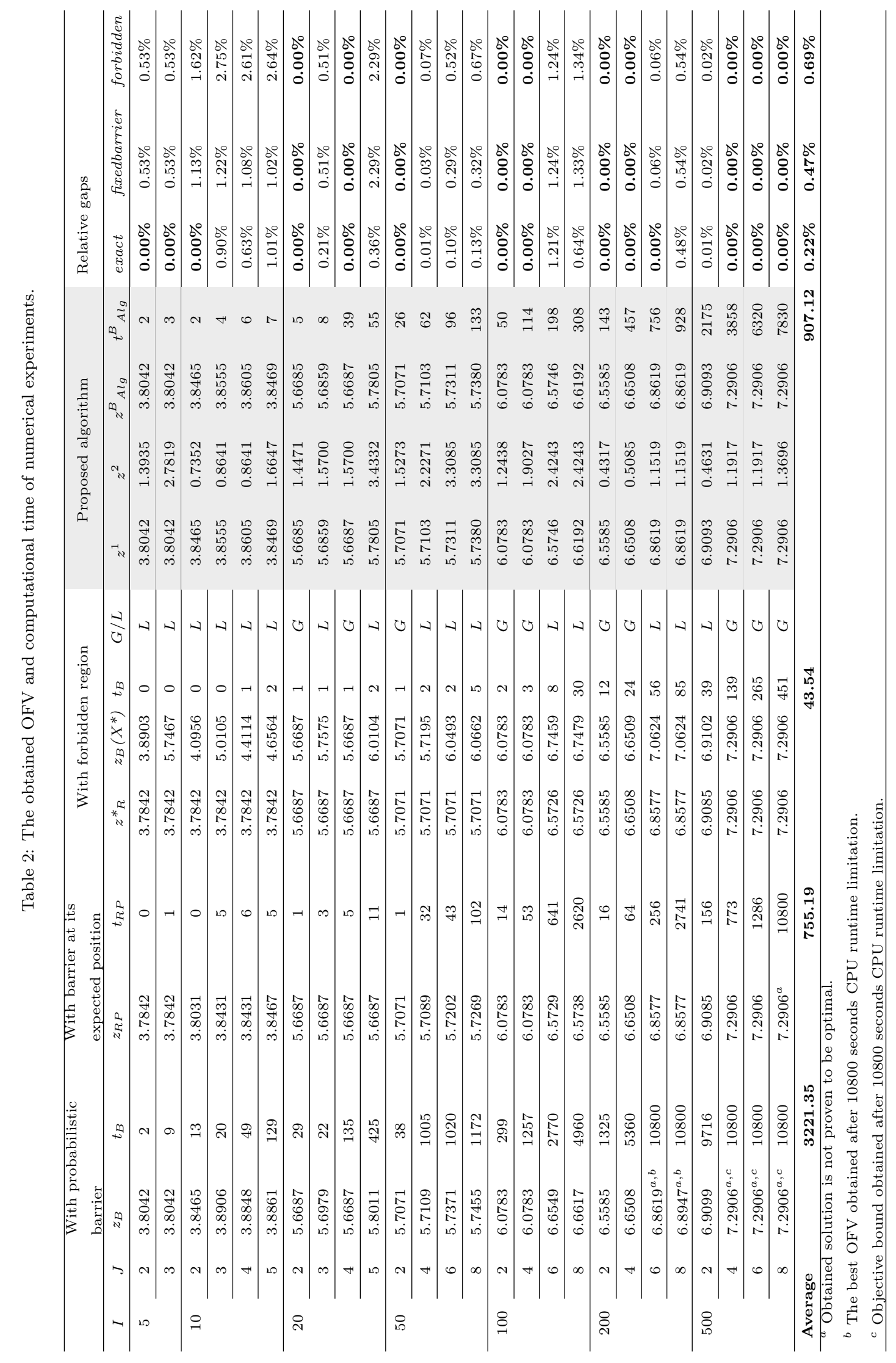


The last line of the Table 2 demonstrates the performance of the algorithms studied in this paper in terms of the algorithm/solver runtime and relative gaps, on average. We see that the proposed algorithm is able to produce, on average, higher quality solutions than those that can be achieved by solving the original with LINGO, and in far less time. Let us recall that the results for the gaps of the proposed algorithm are to be found under the label "Gap-Exact". Similarly, the gaps with the relaxations (Gap-forbidden, Gap-barrier-fixed) indicate that the proposed algorithm is producing solutions that are near-optimal. We next illustrate in Figures 3 and 4 these two metrics by instance size, measured in terms of number of demand points $(I)$ and number of facilities to locate $(J)$. What is clear from the Gaps chart is that the performance of the proposed algorithm is fairly robust with respect to instance size; the relative gap never exceeds $1.21 \%$. And of course there is no reason to believe that the bound yielded by solving the relaxation problem equals the objective function value of the optimal solution. We also see that the bound produced by solving the relaxation that assumes the barrier is fixed at its expected position is typically stronger than the problem with forbidden region, suggesting that it could be a better procedure for producing a lower bound for the location problem with barrier region than was proposed in Klamroth (2001b). We also see from Figure 4 that the time required to solve the original problem with LINGO grows quickly with respect to instance size; for nearly all instances with 200 or more demand points the full 10,800 seconds is required. While the time required by the proposed algorithm also grows, it does so at a much slower rate.

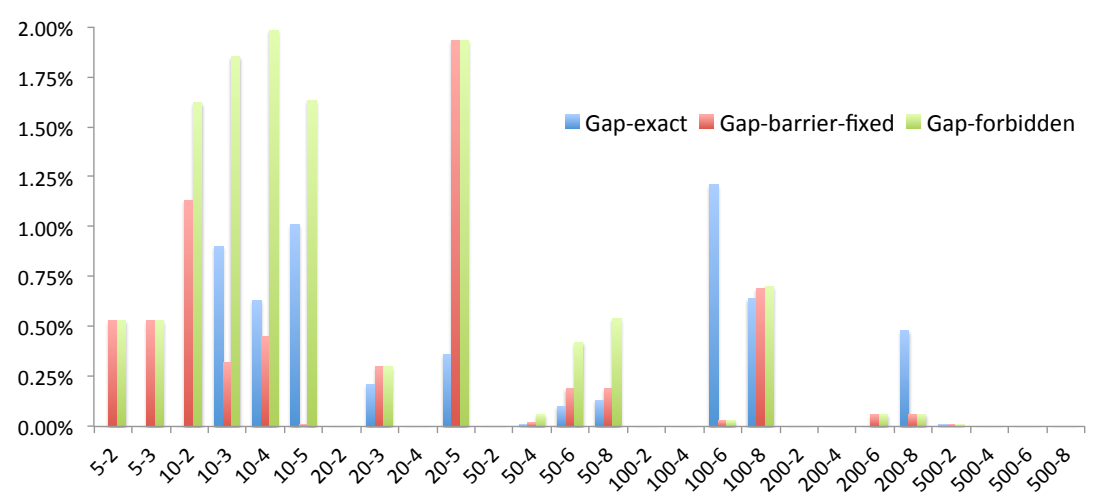

I-J

Figure 3: Gaps by instance size

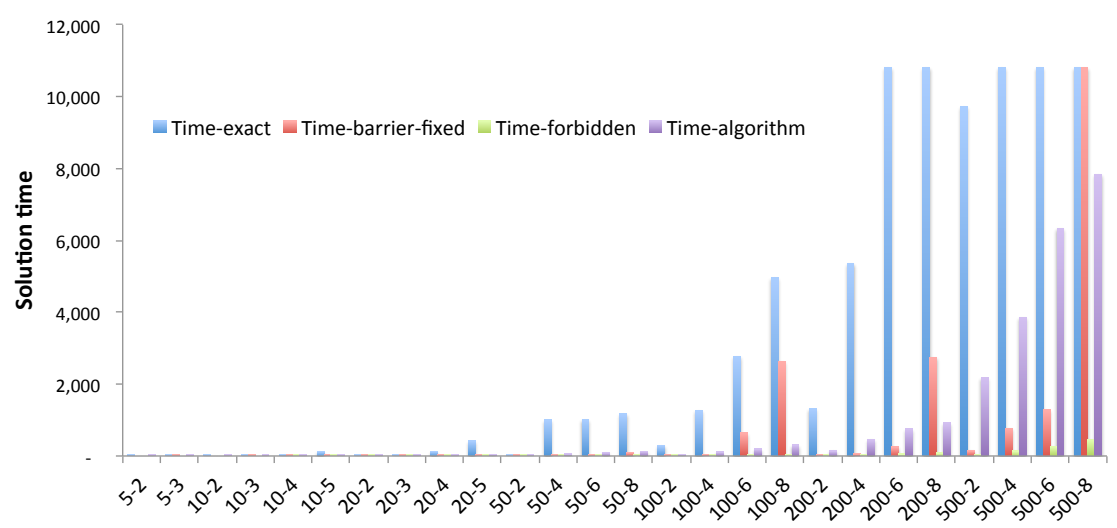

I-J

Figure 4: Solution time by instance size 


\subsection{Case study}

We next illustrate the impact of considering a line barrier when locating facilities with a case study in an urban setting. We choose such a setting as it is one where rectilinear distances on a continuous plane have been shown to provide a suitable approximation for travel distances (Love et al., 1988). We consider the location of seven police departments (as new facilities) in Kingston Upon Thames, England, whose purpose is to respond to calls regarding potential criminal activities in the region. That there are seven departments to be located is based upon the premise that they have different departments for (seven) different types of activities. Kingston contains 16 local areas (Figure 5) that are referred to as wards; in terms of our model, we model these wards as demand points.

That said, as the data regarding reported offences is at the ward level, we model the location of each demand point based on a tansformation of latitude and longitude data regarding postal codes to Cartesian coordinates. We report the resulting cartesian coordinates for each demand point in Table A.1. Specifically, we report Easting, which refers to the horizontal-measured distance (toward the east), and Northing, which refers to the vertical-measured distance (toward the north), We generated "demand" for each location based on the number of reported offences over a 12 month period ending in February, 2013, which we report in Table A.2. We generated demand regarding the interactions between pairs of facilities based on the number of common offences investigated (Branches of the Metropolitan Police Service 2013), which we report in Table A.3.

There is also an overground rail line that runs through Kingston (between Surrbiton and Beverley), and is illustrated by the dashed line in Figure 5a. We model the movement of this train across a road, which blocks traffic, as a probabilistic line barrier. Specifically, the uncertainty regarding the location of this train is modeled with two random variables, with the first representing the location of either the front or back of the train, depending on the direction in which the train is traveling. We presume this random variable (the location of the start of the barrier) is uniformly distributed over the length of the track. We believe that the Uniform probability distribution function can be a reasonable approximation of the randomness of barrier position on its track, as the train departure time over a course of day is approximately uniformly distributed and the speed of the train in the route is almost constant.The second random variable represents the end of the barrier (which can also be either the front or back of the train), and is a function of the length of the train, which we presume is known. We also presume there are no disconnections in the track and that the train repeatedly travels from one end to the next, passing through all stations on the line.

We consider two scenarios in this setting. In the first, the seven departments are located without recognizing the train line. These locations are determined using the classical planar multi-facility location model of Love et al., (1988). In the second, the departments are located with the model proposed in this paper that recognizes the train line. We present in Table 3 information regarding these two solutions. First, we present the ward each department is located in, as well as its Cartesian coordinate. Second, we report the objective function value of each set of locations when evaluated with the proposed model, and thus the probabilistic barrier is recognized. We observe that ignoring the presence of the train increases the expected maximum weighted distance by over $20 \%$.

Finally, we assessed the equitability of the (police department location) solutions obtained by implementing the proposed methods for the considered scenarios to understand how the location decisions and traveled 


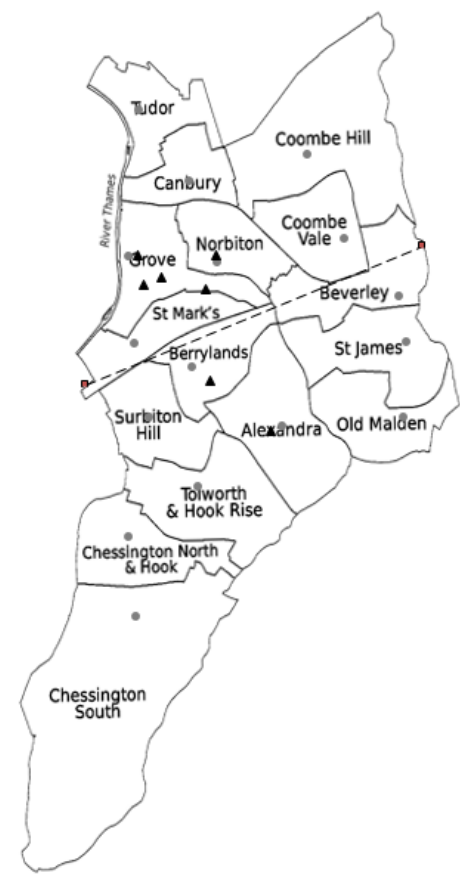

(a) with line barrier

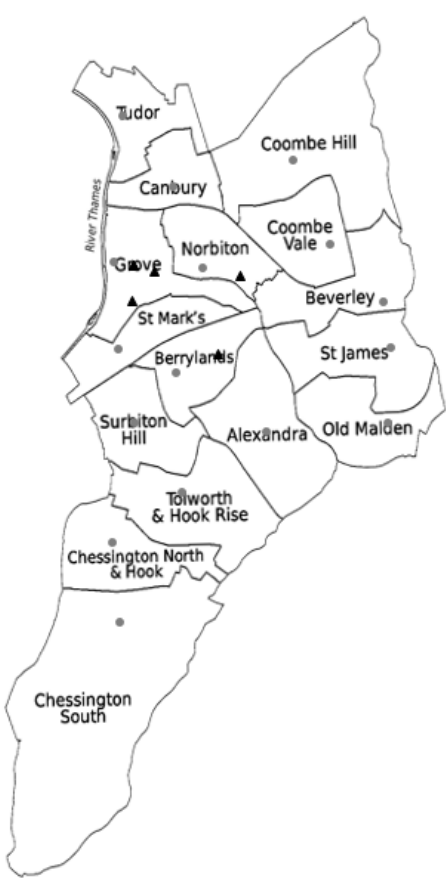

(b) without line barrier

Figure 5: Graphical representation of police departments. Original source of the map: wikimedia

distances would be impacted when responding to an emergency call. Considering the two sets of department locations, we observe that the seven departments are located in different wards in the two solutions. Results suggest that the model that does not recognize the train line locates departments in only three wards (five in Grove and one in Norbiton in the upper side of the train line and only one in Berrylands in the lower side of the train line), whereas the model that does recognize the train line locates departments in five wards (three in Grove, one in St. Marks, and one in Norbiton in the upper side of the train line and one in Berrylands and one in Alexandra in the lower side of the train line). It should be noted that department locations include overlapping. These results are depicted in Figure 5.

We underline that when the model ignores the presence of the train line the maximum expected weighted distance, when locating departments in only three wards, is 267,184 which is nearly $10.2 \%$ less than when the model recognizes the presence of train line and locates them in five wards $(297,470)$. This result may mislead practitioners while offering apparently more interesting solution. However, when the solution produced by the model without barrier (i.e., the scenario in which departments are located in only three wards) is evaluated with our multi-facility model considering the presence of the barrier, we observe that the maximum expected weighted distance is equal to 390,244 , resulting in $23.77 \%$ saving in the weighted travelled distance. This reveals that considering the presence of line barrier in strategic facility location decisions contributes to a remarkable saving in travelled distance, especially in providing equitable services. Finally, we observe that the model that does recognize the train line locates two departments south of the train line whereas the model that does not only locates one. We conclude from this study that explicitly recognizing the line barrier leads to a more 
Table 3: Location of new police departments.

\begin{tabular}{|cccc|ccc|}
\hline & Problem & with & barrier & Problem & without & barrier \\
\hline Police departments & Easting & Northing & Based in & Easting & Northing & Based in \\
\hline Specialist and economic crime & 519,811 & 166,346 & Norbiton & 518,297 & 168,824 & Berrylands \\
Child abuse investigation & 518,228 & 168,454 & Grove & 519,347 & 167,539 & Grove \\
Drugs directorate & 519,130 & 168,884 & Alexandra & 518,297 & 168,824 & Grove \\
Directorate of forensic services & 518,147 & 168,876 & Grove & 518,279 & 168,310 & Grove \\
Homicide and serious crime & 518,450 & 168,560 & Grove & 519,619 & 168,664 & Norbiton \\
Serious and organized crime & 519,048 & 167,072 & Berrylands & 518,544 & 168,736 & Grove \\
Specialist crime \& operations & 519,004 & 168,381 & ST Marks & 518,297 & 168,824 & Grove \\
\hline Expected maximum weighted & & & $\mathbf{2 9 7 , 4 7 0}$ & & & $\mathbf{2 6 7 , 1 8 4}$ \\
distance with barrier & & & & & & \\
\hline
\end{tabular}

robust set of locations for the police departments, which in turn leads to a significant decrease in the maximum expected travel distance.

\section{Conclusion and further research}

This paper studied a multi-facility location problem with an equitability consideration where minimizes the longest distance traveled to demand points (a minimax objective function). We formulated a mixed integer quadratic-constrained programming model of a multiple facility location problem in the presence of a lineshaped barrier and observed that convexity of this model cannot be determined. We then proposed a heuristic that relies on relaxation and split-divide-and-conquer strategy. We computationally studied the performance of the proposed heuristic with an extensive computational study and realized that it produces high-quality solutions in reasonable run-times across all instance sizes. We also presented an efficient lower bound to the current literature. Results reveal that the presence of a barrier has significant impact on the traveled distance to demand points. On the other hand, ignoring the presence of barriers when modeling for strategic facility location may mislead decision-makers to inappropriate solutions. The proposed distance-based model, whose practical application has been discussed in Heizer and Render, (2011), Zanjirani Farahani et al., (2019), and Bélanger et al., (2019), is suitable at the national and regional scope, as it considers some critical factors such as attractiveness of region (e.g. tax incentives and grants related to land cost), ecological regulations (e.g. natural and environmental law), geographical and constructional obstacles (e.g. rivers, parks, utilities, and buildings), and transportation infrastructures, influencing location of several service and emergency facilities (ex: ambulances, fire stations, medical services ...). Deciding upon the location of other facilities that provide emergency services, such as fire stations or hospitals, could also be assisted with this model. Such facilities are often found in urban environments where physical barriers exist.

This paper focused on a rectilinear distance $(p=1)$, which is applicable for urban planning. However, for other settings, such as oceans or deserts, the euclidian measure of distance $(p=2)$ is more applicable. Similarly, in material handling situations, a Tchebychev distance $(p=\infty)$ may be more applicable. This problem can also be extended in the innovative urban context such as electric vehicles (EV) transportation Liu and Wang (2017) 
and seaport yard management for moving the empty and laden container (Park and Seo, 2010). Unlike the problem studied in this paper, in those cases both the $x$ and $y$-coordinates can impact the distance between two points. Similarly, this paper only considered one line barrier. Many settings may involve more than one line barrier and those barriers may have arbitrary shape. In addition, the problem studied in this paper assumed no cuts or disconnections along the barrier route. Clearly, considering a larger geographical area would likely lead to a non-straight, and likely curved, barrier with a properly defined distribution of the line barrier. In this paper, we applied the distance-based location modeling approach for the service and emergency facilities as we focus on a strategic decision-making problem at the regional geographical scope under the existence of barriers. Our model use travel distance as a proxy for travel time. However, fluctuations in travel time (say due to traffic) can impact the service an emergency facility provides. As such, one further extension is to extend the model to also recognize travel times, and that there is uncertainty in those travel times. 


\section{A. Appendix}

\section{A.1. Proofs and mathematical details}

Details of equation (32):

$$
\begin{gathered}
z^{1}=\min _{\left(x_{j}, y_{j}\right)}\left\{\max _{i, j}\left\{w_{i j} \cdot E\left[D_{1}^{B}\left(P_{i}, X_{j}\right)\right]\right\}\right\} \\
=\min _{\left(x_{j}, y_{j}\right)}\left\{\max _{j}\left\{\max _{i}\left\{w_{i j} \cdot E\left[D_{1}^{B}\left(P_{i}, X_{j}\right)\right]\right\}\right\}\right. \\
=\max _{j}\left\{\min _{\left(x_{j}, y_{j}\right)}\left\{\max _{i}\left\{w_{i j} \cdot E\left[D_{1}^{B}\left(P_{i}, X_{j}\right)\right]\right\}\right\}=\max _{j}\left\{z_{j}^{1}\right\}\right.
\end{gathered}
$$



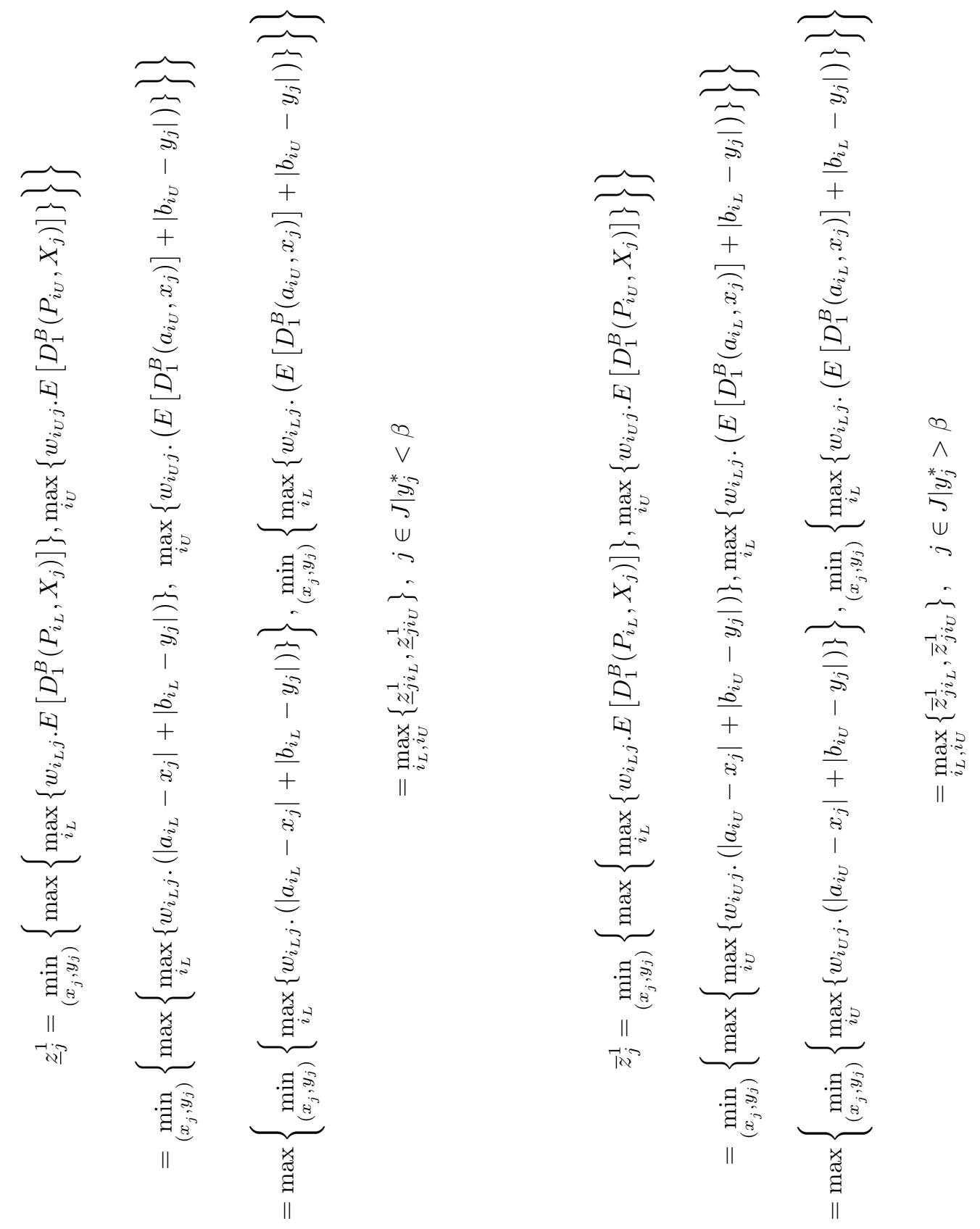

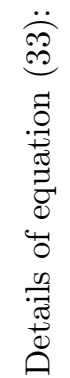

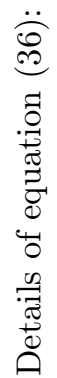




\section{Proof of lemma 1.}

When we fix the line barrier at its expected position, first we should compute the $E\left[X_{s}\right]=\frac{L_{2}+L_{1}}{2}$ that is the middle point of interval $\left(L_{1}, L_{2}\right)$. Since the barrier has length of $l$, the expected value of the start point of the barrier should be $\bar{x}_{s}=\frac{L_{2}+L_{1}}{2}-\frac{l}{2}$ (see Figure A.1). Therefore, the start point happens at $\bar{X}_{s}=\left(\bar{x}_{s}, \beta\right)$ and the end point of the line barrier with the length of $l$ occurs at $\bar{X}_{e}=\left(\bar{x}_{e}, \beta\right)$ where $\bar{x}_{e}=\bar{x}_{s}+l=\frac{L_{2}+L_{1}+l}{2}$.

$$
\bar{x}_{e}=\bar{x}_{s}+l=\frac{L_{2}+L_{1}+l}{2}
$$

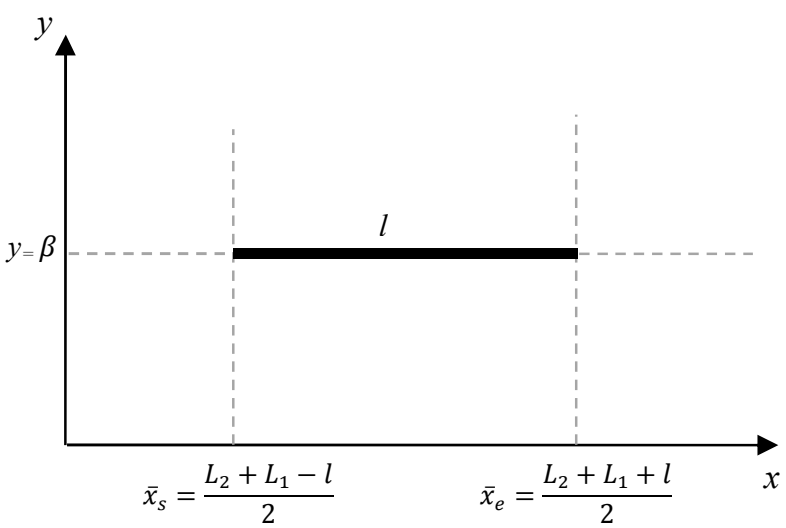

Figure A.1: A line barrier at its expected position.

\section{Proof of lemma 2}

Let us consider the shortest path between $P_{i}$ and $X_{j}$. When $P_{i}$ and $X_{j}$ are located in different half-planes and $\bar{x}_{s} \leq x_{j} \leq \bar{x}_{e}$ and $\bar{x}_{s} \leq a_{i} \leq \bar{x}_{e}$, then the distance between $P_{i}$ and $X_{j}$ becomes the barrier distance and therefore the barrier is in effect. In this case travelling is possible through two ends of the line barrier when barrier conditions are met. The shortest path between $P_{i}$ and $X_{j}$, called $S P_{i j}$, includes one of end points of line barrier either $\bar{X}_{s}$ or $\bar{X}_{e}$, equation (A.5). Note that when the barrier conditions are not met, the barrier distance becomes regular rectilinear distance between $P_{i}$ and $X_{j}$.

$$
\begin{gathered}
S P_{i j}=\min \left\{d_{1}\left(X_{i}, \bar{X}_{s}\right)+d_{1}\left(\bar{X}_{s}, X_{j}\right), d_{1}\left(X_{i}, \bar{X}_{e}\right)+d_{1}\left(\bar{X}_{e}, X_{j}\right)\right\} \\
=\min \left\{\left|a_{i}-\bar{x}_{s}\right|+\left|b_{i}-\beta\right|+\left|\bar{x}_{s}-x_{j}\right|+\left|\beta-y_{j}\right|,\left|a_{i}-\bar{x}_{e}\right|+\left|b_{i}-\beta\right|+\left|\bar{x}_{e}-x_{j}\right|+\left|\beta-y_{j}\right|\right\} \\
=\min \left\{a_{i}+x_{j}-2 \bar{x}_{s}, 2 \bar{x}_{e}-a_{i}-x_{j}\right\}+\left|b_{i}-\beta\right|+\left|\beta-y_{j}\right|, \quad i \in I, j \in J
\end{gathered}
$$

Similarly, the shortest path between $X_{j}$ and $X_{k}$ should be mentioned. If the barrier conditions mentioned above are met, then the barrier is in effect and the barrier distance between $X_{j}$ and $X_{k}$ is the shortest path between $X_{j}$ and $X_{k}$ through two ends of the line barrier, equation (A.6). On the other hand, the barrier distance becomes regular rectilinear distance between new facilities when the barrier conditions are dissatisfied. On the other hand, the barrier distance becomes regular rectilinear distance between new facilities when the barrier conditions are dissatisfied.

$$
\begin{gathered}
S P_{j k}=\min \left\{d_{1}\left(X_{j}, \bar{X}_{s}\right)+d_{1}\left(\bar{X}_{s}, X_{k}\right), d_{1}\left(X_{j}, \bar{X}_{e}\right)+d_{1}\left(\bar{X}_{e}, X_{k}\right)\right\} \\
=\min \left\{\left|x_{j}-\bar{x}_{s}\right|+\left|y_{j}-\beta\right|+\left|\bar{x}_{s}-x_{k}\right|+\left|\beta-y_{k}\right|,\left|x_{j}-\bar{x}_{e}\right|+\left|y_{j}-\beta\right|+\left|\bar{x}_{e}-x_{k}\right|+\left|\beta-y_{k}\right|\right\}
\end{gathered}
$$




$$
=\min \left\{x_{j}+x_{k}-2 \bar{x}_{s}, 2 \bar{x}_{e}-x_{j}-x_{k}\right\}+\left|y_{j}-\beta\right|+\left|\beta-y_{k}\right|, \quad j, k \in J, j<k
$$

A.2. Case study input data

Table A.1: Data for location of wards in Kingston Upon Thames.

\begin{tabular}{|c|c|c|}
\hline \multirow[t]{2}{*}{ Wards (demand points) } & \multicolumn{2}{|c|}{ Cartesian coordinates } \\
\hline & Easting & Northing \\
\hline Alexandra & 519,944 & 166,409 \\
\hline $2 \quad$ Berrylands & 518,818 & 167,270 \\
\hline 3 Beverley & 521,405 & 168,286 \\
\hline Canbury & 518,777 & $169,950.5$ \\
\hline $5 \quad$ Chessington North \& Hook & 518,033 & 164,812 \\
\hline Chessington South & 518,118 & 163,673 \\
\hline $7 \quad$ Coombe Hill & 520,268 & 170,346 \\
\hline 8 Coombe Vale & $520,728.5$ & 169,131 \\
\hline $9 \quad$ Grove & $518,036.5$ & $168,857.5$ \\
\hline 10 Norbiton & 519,141 & 168,788 \\
\hline 11 Old Malden & 521,455 & 166,539 \\
\hline 12 St. James & 521,497 & 167,628 \\
\hline 13 St. Mark's & 518,104 & 167,608 \\
\hline 14 Surbiton Hill & 518,293 & 166,553 \\
\hline 15 Tolworth and Hook Rise & 518,897 & 165,540 \\
\hline 16 Tudor & 518,155 & $1709,69.5$ \\
\hline Start point of the barrier route & 517,500 & 167,000 \\
\hline End point of the barrier route & 521,700 & 169,000 \\
\hline
\end{tabular}


Table A.2: The interactions between the demand points and new police departments in Kingston Upon Thames.

\begin{tabular}{lccccccc}
\hline & \multicolumn{7}{c}{ Police departments } \\
\hline Wards (demand & D1 & D2 & D3 & D4 & D5 & D6 & D7 \\
points) & & & & & & & \\
\hline Tudor & 26.8 & 1.2 & 24.7 & 18.6 & 7.4 & 39.5 & 26.8 \\
\hline Coombe Hill & 35 & 1.5 & 31.5 & 23.2 & 11.5 & 46.9 & 35 \\
\hline Canbury & 34.3 & 1.1 & 31.7 & 22.8 & 10.2 & 50.8 & 34.3 \\
\hline Coombe Vale & 20.4 & 0.6 & 18.9 & 15.9 & 5.7 & 34.6 & 20.4 \\
\hline Norbiton & 50.6 & 2.4 & 48.1 & 35.4 & 21.8 & 71.2 & 50.6 \\
\hline Grove & 36.7 & 0.98 & 35.48 & 11.19 & 8.61 & 40.5 & 36.69 \\
\hline Beverley & 53 & 1.9 & 49.8 & 32.1 & 15.2 & 73.9 & 53 \\
\hline St James & 24.9 & 0.8 & 23 & 21.3 & 9.3 & 39.3 & 24.9 \\
\hline St Marks & 44.4 & 1.3 & 41.8 & 20.6 & 11.9 & 55.1 & 44.4 \\
\hline Berrylands & 20.7 & 1.3 & 19.3 & 15.3 & 8.2 & 30 & 20.7 \\
\hline Surbiton Hill & 22.6 & 1.3 & 21.1 & 15.9 & 7.7 & 33.7 & 22.6 \\
\hline Alexandra & 30.5 & 1.1 & 28 & 18.7 & 8.4 & 43.9 & 30.5 \\
\hline Old Malden & 24.4 & 1.4 & 22.5 & 20.3 & 10.3 & 37.2 & 24.4 \\
\hline Tolworth and Hook Rise & 34.9 & 1.8 & 32 & 28.8 & 13.3 & 54.9 & 34.9 \\
\hline Chessington North and Hook & 27.3 & 0.9 & 25.9 & 21.2 & 9.2 & 47.6 & 27.3 \\
\hline Chessington South & 31 & 1.1 & 28.4 & 21.8 & 12.4 & 42.8 & 31 \\
\hline & & & & & & &
\end{tabular}




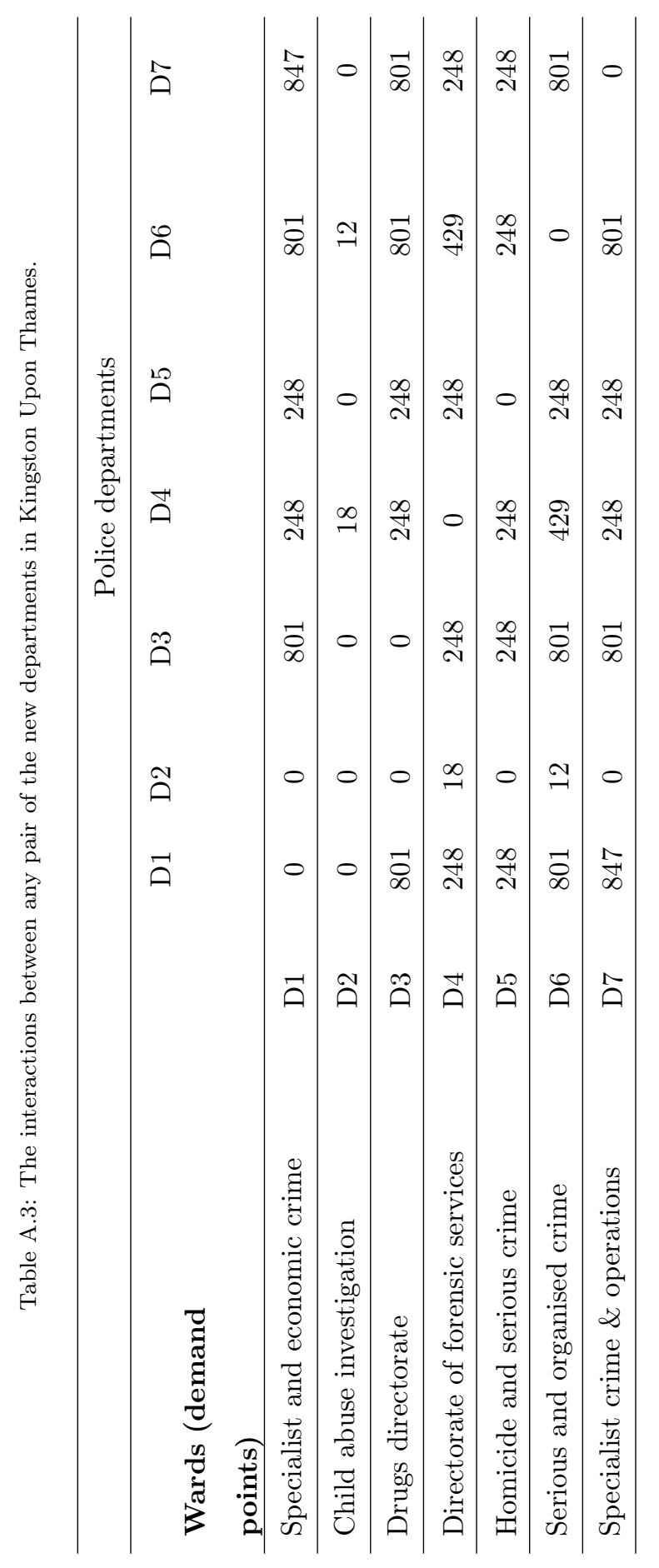




\section{References}

Amiri-Aref, M., N. Javadian, R. Tavakkoli-Moghaddam, A. Baboli. 2013a. A new mathematical model for Weber location problem with a probabilistic polyhedral barrier. International Journal of Production Research 51(20): 6110-6128.

Amiri-Aref, M., N. Javadian, R. Tavakkoli-Moghaddam, A. Baboli, S. Shiripour. 2013b. The center location-dependent relocation problem with a probabilistic line barrier. Applied Soft Computing 13(7): 3380-3391.

Amiri-Aref, M., R.Z. Farahani, N. Javadian, W. Klibi. 2016. A rectilinear distance location-relocation problem with a probabilistic restriction: mathematical modelling and solution approaches. International Journal of Production Research 54(3): 629-646

Aneja, Y.P., M. Parlar. 1994. Algorithms for Weber facility location in the presence of forbidden regions and or barriers to travel. Transportation Science 28(1): 70-76.

Batta, R., A. Ghose, U. Palekar. 1989. Locating facilities on the manhattan metric with arbitrarily shaped barriers and convex forbidden regions. Transportation Science 23(1): 26-36.

Bé langer, V., Ruiz, A., Soriano, P. 2019. Recent optimization models and trends in location, relocation, and dispatching of emergency medical vehicles. European Journal of Operational Research 272(1): 1-23.

Bischoff, M., K. Klamroth. 2007. An efficient solution method for Weber problems with barriers based on genetic algorithms. European Journal of Operational Research 177(1): 22-41.

Bischoff, M., T. Fleischmann, K. Klamroth. 2009. The multi-facility location-allocation problem with polyhedral barriers, Computers and Operations Research 36(5): 1376-1392.

Canbolat, M.S., G.O. Wesolowsky. 2010. The rectilinear distance Weber problem in the presence of a probabilistic line barrier, European Journal of Operational Research 202(1): 114-121.

Dearing, P. M., J.R. Segars. 2002a. Solving rectilinear planar location problems with barriers by a polynomial partitioning. Annals of Operations Research 111(1-4): 111-133.

Dearing, P. M., J.R. Segars. 2002b. An equivalence result for single facility planar location problems with rectilinear distance and barriers. Annals of Operations Research 111(1-4): 89-110.

Dearing, P. M., K. Klamroth, R. Segars, R. 2005. Planar location problems with block distance and barriers. Annals of Operations Research 136:(1) 117-143.

Frieß, L., K. Klamroth, M. Sprau. 2005. A wavefront approach to center location problems with barriers. Annals of Operations Research 136(1): 35-48.

Hakimi, S.L. 1965. Optimal distribution of switching centers in a communications network and some related graph theoretic problem, Operations Research 13: 462-475.

Hamacher, H.W., K. Klamroth. 2000. Planar Weber location problems with barriers and block norms. Annals of Operations Research 96(1-4): 191-208.

Hamacher, H.W., S. Nickel. 1998. Classification of location problems. Location Science. 6: 229-242.

Heizer, J., B. Render. 2011. Principles of Operations Management. 8th Edition, Prentice Hall, Upper Saddle River.

Javadian, N., R. Tavakkoli-Moghaddam, M. Amiri-Aref, M., S. Shiripour. 2014. Two meta-heuristics for a multi-period minisum location-relocation problem with line restriction. International Journal of Advanced Manufacturing Technology 71(5-8): 1033-1048.

Kelachankuttu, H., R. Batta, R. Nagi. 2007. Contour line construction for a new rectangular facility in an existing layout with rectangular departments. European Journal of Operational Research 180(1): 149-162. 
Klamroth, K. 2001a. A reduction result for location problems with polyhedral barriers. European Journal of Operational Research 130(3): 486-497.

Klamroth, K. 2001b. Planar Weber location problems with line barriers. Optimization 49(5-6): 517-527.

Klamroth, K. 2002. Single facility location problems with barriers. Springer Series in Operations Research, (Chapter 4).

Klamroth, K. 2004. Algebraic properties of location problems with one circular barrier. European Journal of Operational Research 154(1): 20-35.

Klamroth, K., M.M. Wiecek. 2002. A bi-objective median location problem with a line barrier. Operations Research 50(4): 670-679.

Love, R.F., J.G. Morris, Wesolowsky, G.O. 1988. Facilities location: models and methods, North-Holland, New York.

Klibi, W., F. Lasalle, A. Martel, S., Ichoua, 2010. The Stochastic Multiperiod Location Transportation Problem. Transportation Science 44: 221-237.

Laporte, G., Dejax, P.J. 1989. Dynamic location routing problems. Journal of Operations Research Society 40(5) 471-482.

Larson, R.C., G. Sadiq. 1983. Facility location with the Manhattan metric in the presence of barriers to travel. Operations Research 31(4): 652-669.

Liu, H., D.Z.W. Wang. 2017. Locating multiple types of charging facilities for battery electric vehicles. Transportation Research Part B: Methodological 103: 30-55.

Lium, A.G., T.G. Crainic., S.W. Wallace, 2009. A Study of Demand Stochasticity in Service Network Design. Transportation Science 43(2): 144-157.

Marcucci, E., V. Gatta, 2014. Behavioral Modeling of Urban Freight Transport. In: Gonzalez-Feliu J., Semet F., Routhier JL. (eds) Sustainable Urban Logistics: Concepts, Methods and Information Systems. EcoProduction (Environmental Issues in Logistics and Manufacturing). Springer, Berlin, Heidelberg.

McLay, L.A., M.E. Mayorga, 2013. A dispatching model for server-to-customer systems that balances efficiency and equity. Manufacturing $\&$ Service Operations Management 15(2): 205-220.

Miyagawa, M. 2010. Distributions of rectilinear deviation distance to visit a facility. European Journal of Operational Research 205(1): 106-112.

Miyagawa, M. 2012. Rectilinear distance to a facility in the presence of a square barrier. Annals of Operations Research 196(1): 443-458.

Miyagawa, M. 2017. Continuous location model of a rectangular barrier facility. TOP 25(1): 95-110.

McGarvey, R.G., T.M. Cavalier. 2003. A global optimal approach to facility location in the presence of forbidden regions. Computers and Industrial Engineering 45(1): 1-15.

Marsh, M.T., D.A. Schilling. 1994. Equity measurement in facility location analysis: A review and framework, European Journal of Operational Research, European Journal of Operational Research 74(1): 1-17.

Nandikonda, P., R. Batta, R. Nagi. 2003. Locating a 1-center on a manhattan plane with arbitrarily shaped barriers. Annals of Operations Research 123(1-4): 157-172.

Oğuz, M., T. Bektaş, J. Bennell, J. Fliege. 2016. A modelling framework for solving restricted planar location problems using phi-objects, Journal of the Operational Research Society 67(8): 1080-1096.

Oğuz, M., T. Bektaş, J. Bennell. 2018. Multicommodity flows and Benders decomposition for restricted continuous location problems, European Journal of Operational Research 266(3): 851-863.

Park, C., J. Seo. 2010. Comparing heuristic algorithms of the planar storage location assignment problem. Transportation Research Part E: Logistics and Transportation Review 46(1): 171-185. 
Pfeiffer, B., K. Klamroth. 2005. Bilinear programming formulations for Weber problems with continuous and network distances. Journal of the Operations Research Society of Japan 48(2): 123-134.

Richard, D., H. Beguin, H. Peeters, D. 1990. The location of fire stations in a rural environment: A case study, Environment and Planning A 22: 39-52.

Sarkar, A., R. Batta, R. Nagi. 2007. Placing a finite size facility with a center objective on a rectangular plane with barriers. European Journal of Operational Research 179(3): 1160-1176.

Savaş, A., R. Batta, R. Nagi. 2002. Finite-size facility placement in the presence of barriers to rectilinear travel. Operations Research 50(6): 1018-1031.

Sayarshad, H.R., J.Y.J. Chow. 2017. Chow, Non-myopic relocation of idle mobility-on-demand vehicles as a dynamic location-allocation-queueing problem. Transportation Research Part E: Logistics and Transportation Review 106(1): 60-77.

Shiripour, S., I. Mahdavi, M. Amiri-Aref, M. Mohammadnia, N. Mahdavi-Amiri. 2012. The multi-facility location problem in the presence of a probabilistic line barrier: a quadratic programming. International Journal of Production Research 50(15): 3988-4008.

Wang, S.J., J. Bhadury, R. Nagi. 2002. Supply facility and input/output point locations in the presence of barriers. Computers and Operations Research 29(6): 685-699.

Zanjirani Farahani, R., S. Fallah, R. Ruiz, S. Hosseini, N. Asgari. 2019. OR models in urban service facility location: A critical review of applications and future developments. European Journal of Operational Research, 276(1): 1-27.

Zhang, Y., L.V. Snyder, T.K. Ralphs, Z. Xue. 2016. The competitive facility location problem under disruption risks. Transportation Research Part E: Logistics and Transportation Review 93(1): 453-473.

Branches of the Metropolitan Police Service. 2013. Retrieved in February 2013 from http://www.met.police.uk/about/ branches/

Latest Crime Figures for Kingston upon Thames. 2013. Retrieved in February 2013 from http://www.met.police.uk/ crimefigures/index $\cdot$ php?borough=vk\&period=year/

UK Postcodes. 2014. Retrieved in January 2014 from http://www.doogal.co.uk/files/postcodes.zip 